[CONTRIBUTION FROM THE LABORATORY OF THE BUREAU OF INTERNAL REVENUE. PUblished by Permission of the Commissioner of Internal Revenue.]

\title{
A STUDY OF THE CHANGES TAKING PLACE IN WHISKEY STORED IN WOOD.
}

BY C. A. Crampton añ L. M. TOLMAN.

Received August 24, 1907 .

This investigation was planned and begun by C. A. Crampton, I. D. Simons and A. B. Adams in the laboratory of the Bureau of Internal Revenue, in 1898 , in order to obtain more definite information concern ing the changes taking place in whiskey when stored in wood, in connection with internal revenue laws relating to the bonding of distilled spirits and the sale of the same under government stamp. The analytical work was completed and manuscript prepared under the direction of L. M. Tolman, with the co-operatian of I. M. Iaw, A. I. Sullivan, E. H. Goodnow and L. B. Forst.

Some work had previously been done in the laboratory by E. Richards upon the maximum quantity of solid matter that could be extracted from oak shavings by proof spirits. ${ }^{1}$

The experiment was conducted with the co-operation of a large number of whiskey distillers, who furnished the material, which was placed in bonded warehouses under the supervision of United States storekeepers.

Thirty-one barrels of new spirits were set aside at that time in as many different warehouses and from as many different distilleries, and a quart sample from each barrel was taken for analysis and sent to the laboratory. These packages were carefully sealed by the officer in charge, to prevent any possible accident to the contents. The gauge of each package was taken, and the condition of the barrel and the warehouse noted.

Each year for eight years during the bonded period, the seals on the packages were broken and a quart was taken for analysis. These samples were all set aside in glass containcrs and a complete chemical ex. amination made in 1906 , after all of the samples from the various packages had been received, except that the determinations of alcohol, solids, color and color soluble in ether were made each year as the samples wer. received.

When the chemical work was begun (1906), we had a series of nine sam ples from each of the thirty-one packages, all of which are exactly the same age, the difference between them being the length of time each had been kept in the barrel. Sample No. I, or the new spirit, had becn kept eight years in glass. Sample No. 2 had been stored one year in wood and seven years in glass. Sample No. 3 had been stored two years in wood and six years in glass, and so on, u1) to the last of the series, which

1 Annual Report, Commissioner of Internal Revenue, for 1880 , page 60. 
had been stored eight years in wood. In the discussion of these samples they will be designated by the length of time they were stored in wood.

The first sample, which was taken from the barrel when it was placed in the warehouse and which represents the fresh distillate, will be spoken of as "new spirit," although, as has been said before, it is of exactly the same age as all of the other san!ples.

The objection to the plan of keeping the samples until all were collected before making the chemical analysis is that we must not take into account the changes taking place after the sample was placed in the bottle; but it seems evident from the chemical analysis of the samples that this factor, the change taking place in the glass, may be neglected, as the results obtained on the new spirits, which had been kept for eight years in glass, showed that practically no change had taken place.

The advantage of this mode of procedure can be readily seen when we consider to what extent the methods of analysis of spirts have changed in the last few years; the results would have been of little comparative value if they had been made by different methods and different analysts. Further, by making all the determinations at one time on the nine samples which make up a series, we are able to detect slight changes that might not have been noticed if the samples had been analyzed eight years apart by different methods and by different analysts. This is especially true in the determination of fusel oil, in which case a uniform method of analysis has made the results very satisfactory, showing the gradual increase taking place from year to year. Fven if the method employed should later be shown to be faulty, the chief value of the results, which is that they are strictly comparable, will not be lost. In the fusel oil work a special effort was made to keep the conditions of analysis absolutely uniform for each period, so as to eliminate the effects of temperature, reagents, etc. All of the nine samples making up the series were started at the same time and carried through to completion by the same analyst. The results obtained by this plan are very remarkable, and would have been entirely impossible with the varying conditions to which they would have been subjected if they had been analyzed year by year. The same plan was followed with all of the other determinations, thus making it possible to show the very small differences which occurred.

\section{Methods of Analysis.}

The methods of analysis used were those of the Association of Official Agricultural Chemists, with the exception of the determination of fusel oil, which was made by the modified Allen-Marquardt method. It was found, however, after a large amount of experimental work which will be published later, that it was necessary with this method to change the 
oxidizing solution used in order to obtain satisfactory results. The following solution was finally adopted: 5 grams of potassium bichromate, $5 \mathrm{cc}$. of concentrated sulphuric acid, the whole made up to $50 \mathrm{cc}$. with water.

It was also found that it was necessary to take smaller amounts of the whiskey for this determination on account of the high content of the fusel oil found in some cases, so that in all these determinations $50 \mathrm{cc}$. of whiskey were used instead of $100 \mathrm{cc}$, as given in the method.

The deternination of the amount of color in the whiskies was made in a half-inch cell by a comparison with the standard glasses of the Lovibond colorimeter, using those of the brewer's scale, and the results are all reported in degrees of this scale referred to the half-inch cell.

The determination of amount of color removed by ether was made by the method of Crampton and Simons,' and results reported as per cent. of color removed.

The determination of the amount of color insoluble in water was made by the method of Walker and Schreiber, ${ }^{2}$ and results reported in per cent. of color insoluble in water.

The following modification of the paraldehyde test was employed: To $5 \mathrm{cc}$. of the whiskey in a test-tube add io cc. of paraldehyde, and shake rigorously; then add absolute alcohol, a few drops at a time, shaking after each addition until the mixture becomes clear, and allow to stand for about ten minutes. A marked turbidity is shown in samples which contain caramel coloring-matter. This turbidity is best observed by holding the test-tube hefore and somewhat below a source of light. None of the samples of this series showed any turbidity by this test, while whiskey containing a very small amount of caramel will give a narked turbidity, showing this to be a reliable positive test for caramel.

The samples were also tested by the Marsh method for caramel color, which is as follows: To $5 \mathrm{cc}$. of the whiskey add ro cc. of the amyl alcohol reagent. as prepared below. Shake vigorously for a few minutes, and allow to settle. With pure whiskey, the lower laver will be perfectly colorless. while if caramel coloring is present, the lower layer will be colored, depending on the amount of caramel present. The reagent is prepared as follows: "lo $100 \mathrm{cc}$ of amyl alcohol add $3 \mathrm{cc}$. of sirupy phosphoric acid and $3 \mathrm{cc}$. of water. Shake to form an emulsion before using. The results obtained on the pure whiskies with this method were very satisfactory, the lower layer being water-white, while with whiskey colored with caramel, the color is concentrated in the lower layer, so that very slight additions of caramel to whiskey can be detected.

1 This Journal, 22, 810-813 (1900).

2 Proc. Assoc. Official Agr. Chemists, Bulletir 99, [i. S. Dept. Agr., Bureau of Chemistry, I), GI. 
The amount of color in the two layers also gives a very satisfactory indication of the amount of color due to the whiskey, and the amount of color which has been added.

\section{Statement of Results.}

The results of analysis are reported, first, in grams per Ioo liters of Ioo proof alcohol, and second, in grams per Ioo liters calculated to the original volume of the whiskey. This second statement of results was made to show how much the increase taking place in the various constituents as the spirits aged was due to the actual increase of these substances in the barrel, and how much was due to the large decrease in volume which takes place at the same time.

For example, the solid matter in solution in whiskey increases each year to a very marked degree, but when we consider the fact that the volume of the spirit in the barrel has diminished about half during the eight-year period of storage, it is seen that a very large amount of the increase in solids is due to this loss in volume, so that the portion of the table where the results are calculated back to the original volume shows the actual increase of each substance in the barrel, while the first portion of the table shows the changes as they would appear in the whiskey when diluted to roo proof.

Unfortunately, the data showing the change in volume taking place each year was not available in most cases, only the volume of the spirits as it was stored and the volume left at the end of the seventh and eighth years having been recorded, but from the results found on the few samples for which the data showing the change in volume from year to year were obtained, we feel justified in plotting the curve of the loss in volume from the three points which were determined, and so estimating the yearly changes. All of the results, however, which are based on these calculated yearly changes have been starred in the table.

The loss of the spirits in volume is not due strictly to evaporation (since it must be remembered that the barrels in which these spirits are stored are made as tight as possible to prevent any leakage), but to the passage of the spirits through the pores of the wood. The barrel acts in many ways like the porous membrane of an osmotic cell and has a very decided selective action on the materials passing through it, as is shown by the large percentage increase by reason of concentration of the ethyl alcohol taking place during storage.

The results indicate that water passes through the wood with much greater rapidity than alcohol under the usual conditions in this cotntry.

The results would also indicate, as shown by chart No. III., that the higher alcohols are completely held back. The same selective action 
is shown with the acids, esters, aldehydes and furfural. Apparently, these substances are left in the barrel by the selective action of the wooden membrane. It is erident that the chenical changes taking place in the spirit in the formation of acids. csters. cte. are dependent on the storage of the spirit in the porous receptacle, as none of these changes occur when spirit is placed in glass, tin, or even barrels, the inside of which has been covered with paraffin or gluc. It is also evident that the condition of the outside of the barrel as to temperature, moisture, etc., will have a decided effect on the rate of osmosis.

\section{Explanation of Terms Used.}

The definitions of the terms "Ryc" and "Bourbon whiskey" are those given in the circular issued by the Committec on Food Standards of the Association of Official Agricultural Chemists.

"Rye whiskey is whiskey in the manufacture of which rye is the principal cereal used, and Bourhon whiskey is whiskey in which Indian corn is the principal cereal userl."

By sweet mash whiskey is meant whiskey in which yeast is used in the fermentation of the mash, and by sour mash whiskey is meant whiskey produced from a mash, the fermentation of which was started by the use of spent beer or slop and barm from tubs previously set and fermented.

The sweet niash fermentation requires much less time for completion, and produces as a ruke, a higher percentage of alcohol.

By the tern "charred package" is meant a barrel, the staves of which have been charred on the inside more or less deeply by the action of fire. The charring of barrels in which whiskey is to be stored is an almost universal practice in this country.

In order to compare the effects of storage in charred and uncharred packages, two samples of whisker, Nos. 2625 and 2637 , were placed in uncharred packages.

"Proof" is the tern used to denote the alcoholic strength of a liquor, and 100 proof is equivalent to 50 per cent by volume of alcohol.

\section{Description of Samples.}

Following are the descriptions of the various samples, giving the kind of whiskey, conditions under which it was stored, composition of the mash used, type of still, with amount and kind of rectification, and yield per bushel of grain, taken from the survey of the distillery, as made by the Bureau of Internal Revenue, together with a discussion of the effect of these varying conditions as shown by analytical results:

\section{Whiskey Aged in Charred Packages.}

Nis. 2.598 (See 'Table I): Sweet mash, rye whiskey, entered into warehouse Jannury 6,1898 , in a new, charred, oak barrel; warehouse, dry and above ground; average 
temperature, $80^{\circ}$ to $85^{\circ} \mathrm{F}$. Composition of the mash: malt, I,064 pounds; and rye, $8,45^{6}$ pounds. Distilled from copper still, capacity $I, I 25$ gallons; redistilled in a second still of the same capacity. The spirit is run directly from the still to the cisternroom without any form of rectification. Yield per bushel of grain, 4 gallons of proof spirits. The results show the effect of aging in a heated warehouse, there being a large loss in volume, increase in proof and a great amount of acids and esters formed. The product shows a very high flavor.

No. 2599 (See Table I): Sweet mash, rye whiskey, entered into bonded warehouse January $I$, 1898, in a new, charred, oak barrel; warehouse, dry and above ground, package was on the fourth floor; average temperature, $85^{\circ} \mathrm{F}$. Composition of the mash: malt, 3,696 pounds; rye, 15,008 pounds. Distilled in three-chambered still, with a doubler, all of copper. The spirit is taken directly from the still to the cisternroom without rectification. Yield, 4 gallons of proof spirits to the bushel of grain. This package shows a very great loss of volume on storage, 58 per cent. in six years, and 61 per cent. in seven years, when the package was taken from storage and sold. The proof also increased to an extraordinary degree, changing from IO2 at the beginning to $14 \mathrm{I}$ at the end of seven years. These changes are greatly hastened by the high temperature of the warehouse, $85^{\circ} \mathrm{F}$. the year round. The matured product shows a very fine flavor and taste.

No. 2600 (see Table I): Sweet mash, rye whiskey, entered into warehouse January 1,1898 , in a new, charred, white oak barrel, free of sap; warehouse, dry and above ground; average temperature in winter is $77^{\circ}$, in summer $85^{\circ} \mathrm{F}$. Composition of the mash: 997 pounds of malt; $x_{4}, 04 \mathrm{I}$ pounds of rye. Distilled in a large wooden still of 10,000 gallons capacity, with a copper doubler of 2,000 gallons capacity. The spirit goes directly from the doubler to the cistern-room, without any form of rectification. Yield per bushel of grain, 4 gallons of proof spirit. This represents the old style whiskey distilled in the large wooden still.

No. 260I (see Table II): Sweet mash, rye whiskey, entered into bonded warehouse January 3, I898, in a new, charred, white oak barrel; warehouse, dry and above ground, with an average temperature in the winter of $80^{\circ}$, in the summer of $90^{\circ}$. Composition of the mash: malt, 2,844 pounds; rye, 14,032 pounds; molasses, 30 gallons. Distilled from a large three-chambered copper beer still, heated by direct steam; capacity, 5,4I2 gallons, with a small copper still heated with a steam coil, having a capacity of $\mathrm{r}, 200$ gallons, which is used as a doubler. The spirit is taken directly from the still to the cistern-room without rectification. Yield, 4 gallons of proof spirit per bushel of grain. This package shows a large loss in volume and a high temperature in warehouse, $80^{\circ}$ in winter and $90^{\circ}$ in the summer. This is also the only sample in which molasses or any other form of sugar has been added to the mash.

No. 2602 (see Table II): Sweet mash, rye whiskey, entered in warehouse January I, I898, in a new, charred, white oak barrel; warehouse, brick, steam-heated, dry and above ground; average temperature during the first year $85^{\circ}$; afterwards, average temperature $70^{\circ}$ Composition of the mash: 3,625 pounds of barley malt; 24,375 pounds Michigan rye. Distilled in a three-chambered copper still, capacity 7,534 gallons, and a copper doubler, capacity r,ooo gallons. The spirits are run from doubler to condenser and then direct to cistern-room, with no other form of rectification. Yield, 4 gallons of proof spirits per bushel of grain.

No. 2603 (see Table II): Sweet mash, rye whiskey, entered into warehouse January I, 1898, in a new, charred, oak barrel; warehouse, brick, dry, and above ground, with an average temperature of $82^{\circ}$. Composition of the mash: malt, 2,520 pounds; I 2,600 pounds rye. Distilled in a large wooden still, capacity 3,554 gallons, heated 
by live stean, with a copper doubler, 66I gallons capacity. High wines are diluted to $80^{\circ}$ proof, and redistilled in a copper still of $1,05^{\circ}$ gallons capacity, heated with a steam coil. Spirit from high wine still, goes directly to the cistern-room without receiving any treatment. Field per bushel of grain, 4.30 gallons of proof spirit.

No. 2604 (see Table III): Sweet mash, rye whiskey, entered into bonded warehouse January 1,1898 , in a new, fully charred, white oak barrel; warehouse, dry and above ground; average temperature, $80^{\circ}$. Composition of the mash: malt, 0,552 pounds; rye, 22,848 pounds. Distilled in a three-chambered copper beer still, which is charged at intervals. The low wines are redistilled in a copper doubling still, capacity 1,389 gallons. The spirit is run directly from the still to the cistern-room without any form of rectification. Yield, 4 gallons of proof spirit to the bushel of grain.

No. 2605 (see Table III): Sweet mash, rye whiskey, entered into bonded warehouse January $\mathrm{I}, \mathrm{s} 8 \mathrm{~g} 8$, in a new, charred, white oak barrel; warehouse, brick, dry and above ground; average temperature, $70^{\circ}$. Composition of the mash: malt, 2,072 pounds; rye, I2,040 pounds. Distilled in two wooden stills, one of a capacity of 3,827 gallons, the other of 2,625 gallons. Yield, 4 gallons of proof spirits to one bushel of grain. This is an old style "pot still" whiskey, distilled in two large wooden stills, and shows by the anount of fusel oil present that the spirits are not rectified to so great an extent as occurs with the other styles of stills in use.

No. 2606 (see Table III): Sweet mash, rye whiskey, entered into warehouse January 1, I 898, in a charred, white oak barrel; warehouse, brick, dry and above ground: average temperature, $75^{\circ}$. Composition of the mash: malt, 1,680 pounds; rye, 9,520 pounds. Distilled in a large wooden still, capacity 9,695 gallons, with a doubler, capacity 6,060 gallons. The spirit is taken directly from the doubler to the cisternroom without rectification. Vield per bushel of grain, 4 gallons of proof spirit.

No. 2607 (see Table IV): Sweet mash, rye whiskey, entered into warehouse Ianuary 3,1898 , in a new, charred, oak barrel; warehouse, brick, dry and above ground; average temperature, $75^{\circ}$. Composition of the mash: malt, I, 260 pounds; rye, 7,140 pounds. Distilled from two wooden pot stills, one with a capacity of 2,158 gallons and one with a capacity of 1,754 gallons, and a copper doubler. The spirit runs directly from the still to the cistern-room, no leach tubes or rectifiers being used in the process. The yield is + gallons to the bushel of grain.

No. 26 ro (see Table IV): Sweet mash, rye whiskey, entered into warehouse January 1,1898 , in at new, charred, white oak barrel; warehouse, brick, dry and above ground, temperature varying between $60^{\circ}$ and $75^{\circ}$. Composition of the mash: malt, 6,720 pounds; rye, 38,080 pounds. Distilled from a copper still, capacity 1,900 gallons; low wine still, capacity 1,500 gallons, with a doubler. The spirit from the stills is run directly to the cistern-room without treatment. Yield per bushel of grain, 4 gallons of proof spirit.

No. 2611 (see l'able IV): Sweet mash whiskey in which corn has been used in excess, entered into bonded warehouse January 1,1898 , in a new, charred package, third floor of warehouse; average temperature, winter $40^{\circ}$, summer $70^{\circ}$. Composition of the mash: malt, 5,376 pounds; rye, 31,360 pounds; corn, 41,664 pounds. Two large wooden stills were used. The spirit from the still is doubled in copper doublers, but does not run through charcoal leach tubes going directly from still to cisternroom. Yield, + gallons to bushel.

No. 2612 (see Table V): Sweet nash, Bourbon whiskey, entered into bonded warehouse Jannary I, Itigs, in a new, heavily charred, oak barrel; warehouse, dry and above ground; average temperature in the winter is $45^{\circ}$, and in the summer $80^{\circ}$. Cumposition of the mash: malt, 8,960 pounds; rye, 1,792 pounds; corn, 78,848 
pounds. Distilled in a continuous, copper still with a column and doubler, receiving no rectification after leaving the still. Yield, 4.5 gallons of proof spirits per bushel of grain. This sample is evidently very highly rectified in the process of distillation. As shown by the analysis, it contains only a trace of fusel oil and suffered very small loss of volume in aging, perhaps due to being stored in an unheated warehouse. The sample was practically cologne spirits aged in a charred package. The taste of the aged whiskey was very little different from that of new spirits, although the odor was much like that of whiskies stored in wood. Even the eight-year old sample had the taste of spirits, differing in a marked degree in taste from the products distilled in wooden stills in which there was little rectification.

No. 2613 (see Table V): Sour mash, rye whiskey, entered into bonded warehouse January I, I898, in a new, charred, oak barrel ; warehouse, dry and above ground; average temperature, $70^{\circ}$. Composition of the mash: malt, 2,576 pounds; rye, 29,344 pounds. Distilled in a three-chambered still with a doubler, capacity of the still 6,665 gallons, capacity of the doubler $1,77^{\circ}$ gallons. The spirit from the doubler is passed through a charcoal filter before being taken to the cistern-room. Yield, 4.5 gallons to one bushel of grain. This was the only sample of rye whiskey made by the sour mash process, and the only one which was rectified by passing through charcoal filters.

No. 2614 (see Table V): Sweet mash, rye whiskey, entered into bonded warehouse January 15,1898 , in a new, charred, white oak barrel; warehouse, new, dry and above ground; average temperature, $62^{\circ}$. Composition of the mash: rye, 5,950 pounds; malt, 1,050 pounds. Distilled in a large wooden still, capacity 7,766 gallons, with a copper doubling still of 1,065 gallons capacity. The spirits are run direct from the still to the cistern-room without rectification. Yield, 4 gallons of proof spirit to one bushel of grain. This sample shows to a remarkable degree the fact that spirit kept in bottles does not undergo any change. The new spirit having been kept over eight years in bottles was alkaline to phenolphthalein, due doubtless to the water used in diluting the spirit to proof, as compared with the same spirit which had been eight years in wood, which contained 91.9 grams of acid per roo liters. This shows that the chemical action taking place is largely brought about by the wood and char in the barrel, which act as catalyzing agents.

No. 2623 (see Table VI): Sweet mash, rye whiskey, entered into warehouse January 28,1898 , in a new, charred, white oak barrel; warehouse, brick, dry and above ground; average temperature in winter $65^{\circ}$, in summer $70^{\circ}$. Composition of the mash: rye and barley malt. Distilled in a large three-chambered wooden still, capacity 15,177 gallons, with a copper doubler of 1, I 16 gallons capacity; redistilled, or run through a copper still before going to the cistern-room. Yield per bushel of grain, 4.5 gallons of proof spirit. Odor and flavor very good in matured goods; body, very light.

No. 2608 (see Table VII): Sour mash, Bourbon whiskey, entered into bonded warehouse January I, 1898 , in a new, charred, white oak barrel; warehouse, dry and above ground; average temperature, $70^{\circ}$. Composition of the mash: malt, 2,240 pounds; rye, I,400 pounds; corn, 16,500 pounds. Distilled in two copper stills, capacity 2,303 gallons and 2,315 gallons, with two doublers of 1,089 gallons capacity each. Yield, 4 gallons to the bushel of grain.

No. 2609 (see Table VII): Sour mash, Bourbon whiskey, entered into warehouse January I, I898, in a new, charred, white oak barrel; warehouse, new, brick, dry and above ground; average temperature in winter $36^{\circ}$, in summer $80^{\circ}$. Composition of the mash: malt, 2,800 pounds; rve, 4,144 pounds; corn, 21,056 pounds 
Distilled in a pot still of 2,000 gallons capacity, with a doubler of $\mathrm{r}, 000$ gallons capacity, both of copper. 'The spirit is taken directly from the doubler to the cistern-room without any form of rectification. Yield, 4.5 gallons of proof spirit to the bushel of grain. This sample shows a comparatively small loss of volume during the eight years' storage, due to the fact that the warehouse is unheated.

No. 2635 (see Table VII): Sour mash, Bourbon whiskey, entered into bonded warehouse February 7 , r 898 , in a well-seasoned, charred, white oak barrel, stored on the third floor of a dry, brick warehouse; average temperature, $70^{\circ}$ to $80^{\circ}$. The mash is distilled from double pot stills, each of 2,000 gallons capacity, and with a doubler of 900 gallons capacity. The spirit is taken directly from the stills to the cisternroom without passing through any form of rectification. Yield per bushel of grain, 3.75 gallons of proof spirit. Composition of the mash: rye, 2,688 pounds; malt, 4, I 44 pounds; corn, 30,800 pounds. The low fusel oil indicates that in the doubling of the whiskey a great deal of rectification has taken place. Distilled with wood fire under doubler.

No. 2636 (see Table VIII): Sweet mash, Bourbon whiskey, entered into bonded warehouse February I I, I 898 , in a new, charred, oak barrel; warehouse, dry and above ground; the water used for reducing proof is distilled; average temperature in the summer $85^{\circ}$, in the winter $70^{\circ}$. Composition of the mash: malt, 1,288 pounds; rye, I,400 pounds; corn, 9,744 pounds. Distilled in a chambered still, capacity 4,500 gallons. The spirit runs directly to the cistern-room, no leach tubes or rectifiers being used. Yield, 4.5 gallons to the bushel of grain.

No. 2637 (see Table VIII): Sweet masl, Bourbon whiskey, entered into bonded warehouse February II, I898, in a new, charred, white oak barrel; warehouse, brick, dry and above ground; average temperature in the summer $80^{\circ}$, in the winter $48^{\circ}$. Composition of the mash: malt, 6,496 pounds; rye, 8,288 pounds; corn, 27,776 pounds. Distilled in a pot still of 3,525 gallons capacity, with a doubler of $I, 395$ gallons capacity. The spirit runs directly from the still to the cistern-room without rectification. Yield, 4.5 gallons of proof spirit to the bushel of grain.

No. 2639 (see Table VIII): Sour mash, Bourbon whiskey, entered into bonded warehouse February 8,1898 , in a new, charred, white oak barrel; warehouse, brick, dry and above ground; average temperature in summer $60^{\circ}-65^{\circ}$, in winter $55^{\circ}$ $60^{\circ}$. Composition of the mash: malt, 4,704 pounds; rye, 9,408 pounds; corn, 32,704 pounds. Distilled with a pot still, capacity 4,526 gallons, and a doubler, capacity I,600 gallons. Yield, 4 gallons of proof spirit per bushel of grain. This sample shows the effect of the temperature of the warehouse in loss of spirits in storage, also the small increase in proof as compared with the samples that show high losses of volume.

No. 2684 (see Table IX): Sour mash, Bourbon whiskey, entered into bonded warehouse March 31, I898, in a new, charred, oak barrel; warehouse, frame, dry and above ground; average temperature in summer $70^{\circ}$, in winter $40^{\circ}$. Composition of the mash: malt, 2,800 pounds; rye, I,680 pounds; corn, 15,680 pounds, Distilled in a large pot still, capacity 8,000 gallons, with a doubler of 1,400 gallons. 'The spirit is taken directly from the doubler to the cistern-room without rectification of any kind. Yield, 4 gallons of proof spirit to one bushel of grain.

No. 2683 (see Table IX): Sour mash, corn whiskey, entered into bonded warehouse April 9, 1898, in a new, charred, oak barrel; warchouse, brick and heated by steam. Average temperature in summer $75^{\circ}$, in winter $40^{\circ}$. Composition of the mash: malt, I, I 76 pounds; rye, 840 pounds; corn, 9,184 pounds. Distilled with a three-chambered still to $60^{\circ}$ proof, then doubled in a pot still heated by fire. The 
spirits from the doubler are run through charcoal leach tubes before going to the cistern-room. Yield, 4.25 gallons per bushel of grain.

No. 2644 (see Table IX): Sour mash, Bourbon whiskey, entered into bonded warehouse January 20,1898 , in a new, charred, white oak barrel; warehouse, iron-clad, dry and above ground, not artificially heated. Composition of the mash: malt, 6I6 pounds; rye, 924 pounds; corn, 7,952 pounds. Distilled in a pot still with a capacity of 3,374 gallons, and with a doubler, capacity of 1,500 gallons. The spirt is run directly into the cistern-room, without any form of rectification. Yield, 4.5 gallons to one bushel of grain. This whiskey shows a slight loss of volume, as compared with the other whiskies, and at the same time the changes taking place in the package have been much less active, as shown by the slight change in proof and relatively small amounts of color, solids, acids, and esters. This emphasizes the fact that reactions taking place in the aging of the product are due largely to the temperature and the rate of osmosis, or the passage of the spirit through the wood.

No. 2685 (see Table X): Sour mash, Bourbon whiskey, entered into bonded warehouse April 9, 1898 , in a new heavily charred white oak barrel; warehouse, dry and above ground. Average temperature in the summer is $80^{\circ}$, in the winter $45^{\circ}$. Composition of the mash: malt, $I, 960$ pounds; rye, 7,840 pounds; corn, 5,600 pounds. Entered as a Bourbon whiskey, but contains rye in excess, as shown by the composition of the mash. Distilled in a continuous, copper, beer still, the low wines from which are doubled in a copper doubler and are not rectified or refined in any way after leaving the doubler, and before reaching the cistern-room. Yield, 3.5 gallons of proof spirits per bushel of grain.

No. 2686 (see Table X): Sour mash, Bourbon whiskey, entered in bonded warehouse April I2, I898, in a new, heavily charred, oak barrel; warehouse, dry and heated by steam. Average temperature in summer $70^{\circ}$, in winter $65^{\circ}$. Composition of the mash: malt, 6,272 pounds; rye, 5,432 pounds; corn, 36,512 pounds. Distilled in a beer still 23 feet high, with a doubler, the column being about the same height as the beer still. Capacity of the still 3,480 gallons; capacity of the doublers 1,440 gallons. The spirit is taken directly from the doubler to the cistern-room without any form of rectification.

No. 2689 (see Table X): Sour mash, corn whiskey, entered into bonded warehouse April 4, 1898, in a new, charred, oak barrel; warehouse, dry and above ground; samples on the top shelf; average temperature in summer $72^{\circ}$, in winter $42^{\circ}$. Composition of the mash: malt, 336 pounds; rye, 224 pounds; corn, 2,968 pounds. Distilled in a copper still with a column. The low wines are redistilled in a doubler which is practically a large pot-still. The beer still has a capacity of $I, 200$ gallons and the doubler of 800 gallons. The spirit from the doubler is leached through tall, narrow vats, into which powdered charcoal has been packed. The whiskey slowly percolates through this charcoal, taking several days to reach the cistern-room. The charcoal used in this leaching is made by burning sugar-maple wood in the open air. The yield per bushel of grain is 3.75 gallons of proof spirits. The very low content of fusel oil shows that considerable rectification has taken place. The high ash of the product indicates that in leaching through the charcoal large quantities of mineral matter are dissolved. The high amount of solids is due largely to the ash. The flavor is good and also the aroma.

No. 2689 A (see Table XI): Sour mash, corn whiskey, entered into bonded warehouse April 9, 1898, in a new, charred, oak barrel. Composition of the mash: malt, 672 pounds; rye, 728 pounds; corn, 5,824 pounds. Distilled in a chambered still with a large doubler, 4,00o gallons capacity. The spirits from the doubler is run through leach tubes, filled with charcoal. Yield, 4.5 gallons per bushel of grain.

Following are the tables of analyses I to XII: 


\section{范}

121

tก! ग19nโOSt! म0I00

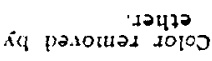

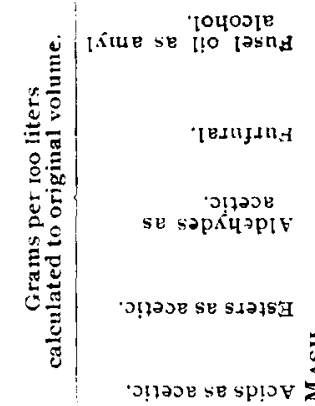

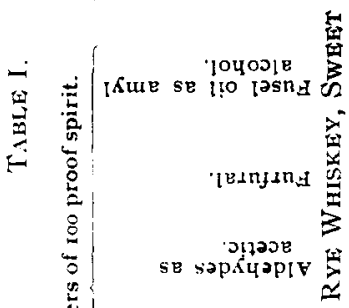

8

岌

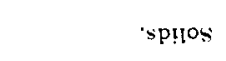

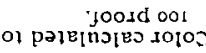

\section{Joosd}

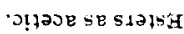

o!tase se sptop

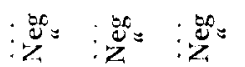

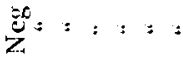

$00: 0: 0 \%$

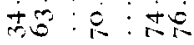

$00+00000$

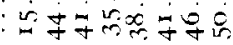

\section{$000.0 \mathrm{ir}$}

ir ir $x: 0$

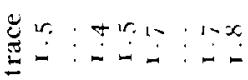

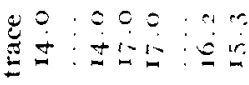

$$
\because: \approx r \leq x)
$$

\section{$\overrightarrow{0}: x \sin x: \dot{0}$}

$\frac{1}{2}$

空

तir. - $\theta x=$. 00

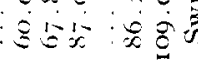

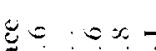
$+\infty$

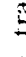

$\& r . \approx x=$

$\approx x=$

$x$

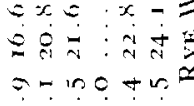
$: \frac{1}{2}$

$\because 10+10,100 x$ ro

तฏ

$000=00000$

ปむல

$01-00-10+7$

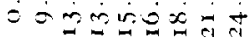

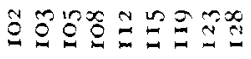

$: \frac{S}{20}=::::$

$\frac{\infty}{7}=:=:$

$0:=: 000$

$x: g: s \approx 0$

$\because 000000$

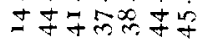

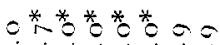

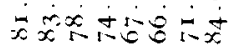

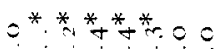

a. a t a d a d a d a

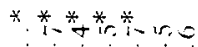

- $x<x-x$ in

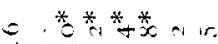

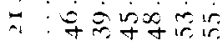

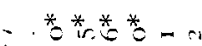

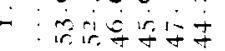

$-+0 x 000=$

$\hat{\sigma} \dot{x} \vec{x} \approx \hat{\theta}=\hat{\theta}$ $\Xi:=-+\sigma=1$.

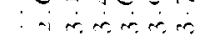

$\because: 000000$

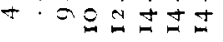

$=$ tatom

- ir i is 0

$\therefore-m+\cdots+\cdots$

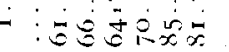

$\because 0000=00$

O

$0000-4 a \mathrm{ar}$

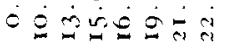

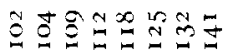

$: \sum^{\circ}=0:=$

$: \frac{d}{Z}=:=::=$

$: 0 \cdot 0 \cdot 0 \cdot 00$

$: \pi: 8: 8:$ :

- r. o o o o o o

-

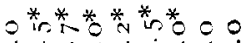

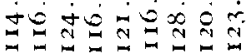

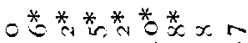

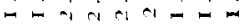

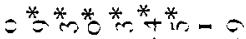

$0 \approx-x \leq 0 \leq 0$

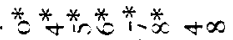

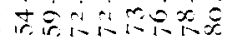

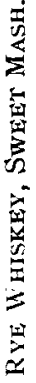

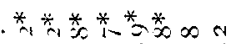
: क्ष $0=0000000$ = $\infty 1-2+1,0+1-0$

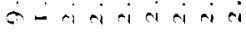

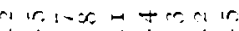

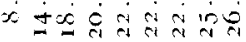
$\pi, 0-x$ 1 00 : in $0 \dot{0}=000$ - acos ancoro : in 00000000 $\because \pi$ ir

0 ax no r.sond

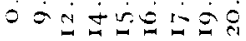

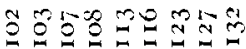

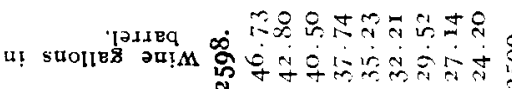
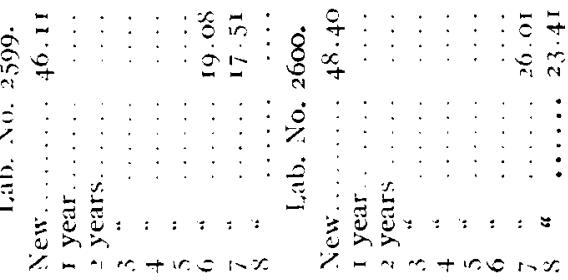
苍

.дرea $0.0: 0: 0: 00$

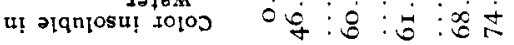
$0 m+000000$ кq pasomas دolos

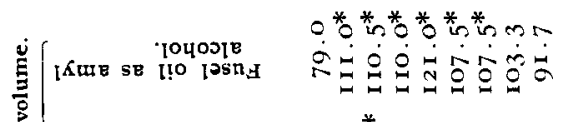

. כịวE SE SIOjST

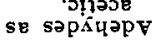

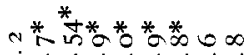

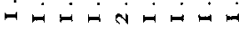

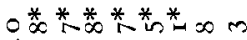

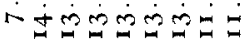

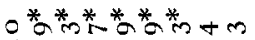

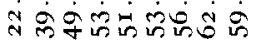
峞

. จฺวग8 sB sp!ov

空

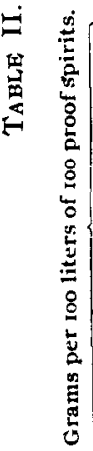

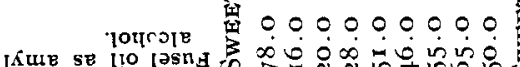
SE lio [asna 年

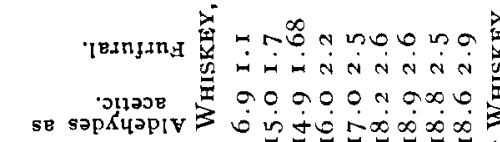
opjasesesiatst त में

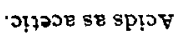

mWMNOON N

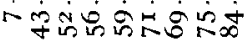
o. 0000000 spllos

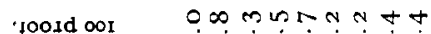

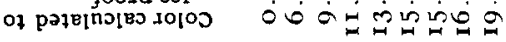
- O. O0.7M0

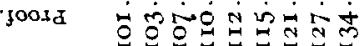

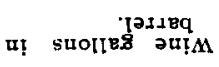

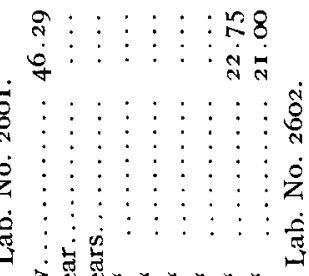

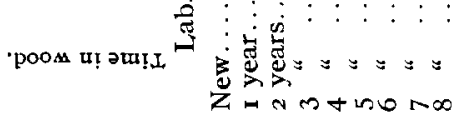

$: \frac{80}{Z}=z=z=5$

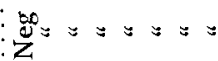

$00: 0 \cdot 0 \cdot 00$ c - $0+000000$ -

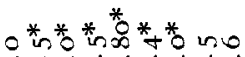

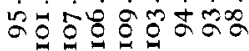

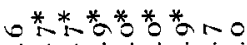

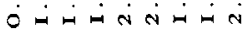
N ${ }_{\infty}^{*} \ddot{t}^{*}+* * * * * * \infty$ लि $\dot{0} \dot{0} \dot{0} \dot{0} \dot{0} \dot{0} \infty$

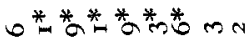

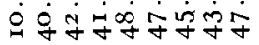

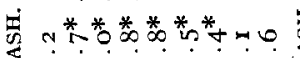

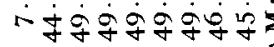
onm $4 m+\infty \infty$ n

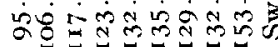

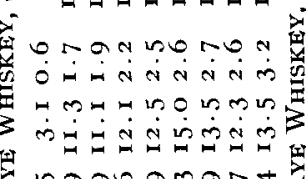
nagogmant

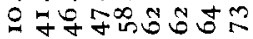
MーNRONー

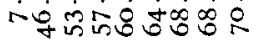
000000000

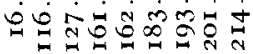

ontionamand 0ெ旦 - No 000000

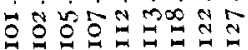

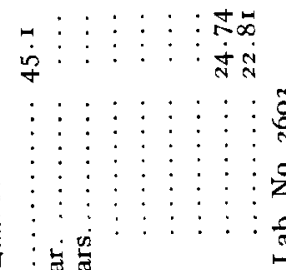
窟焉: = = = Z

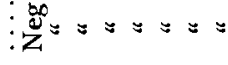

$: \frac{80}{Z}=z=z=5$

○ी:?:?:0\% ० ด. 9.0.0.0.0 -

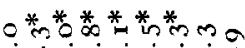

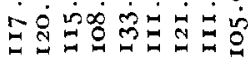

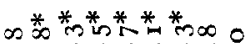

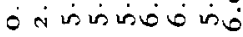

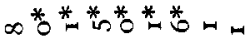

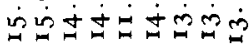

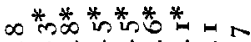
은

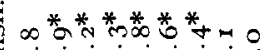

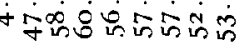
on

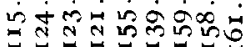
कantrmmun

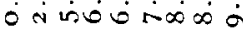
o 0 nm nomo

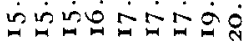

0HVNMMNm मिं H n muomon nao

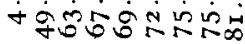
- 00000000 की A $2 \pi$ 年 숭용요

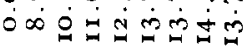
- o. o. 0000

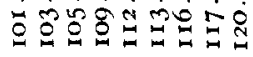

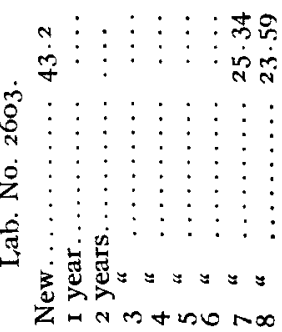




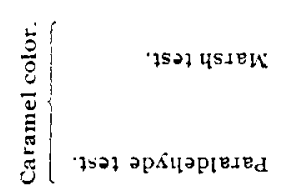

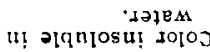

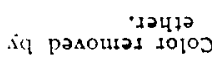

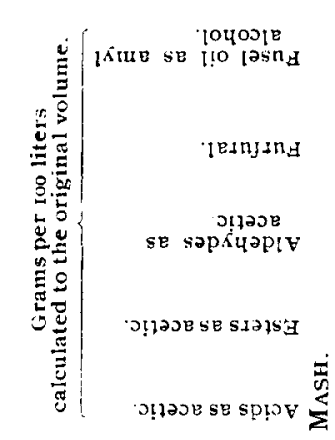

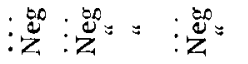

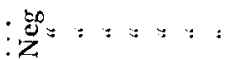

$: 0: 0: 0: 00$ $: \pi: 8:$ in: $: 00$ - Med o o o o o

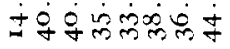

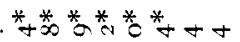
:

: 苞: * *艹

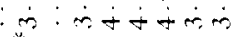

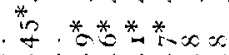

$\therefore: \dot{0} \dot{0} \dot{0} \dot{0}$

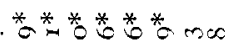
im+osis ******

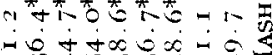
$\rightarrow+\sin$ in ir. o o o w a e w m

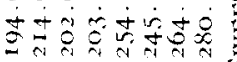
m $10 x-+\infty-$ $\therefore \therefore$ को ir ir $\dot{0}$ ir.

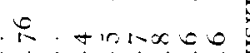
$\therefore$ ain a nolomat m=

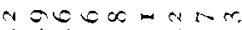

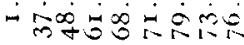
000000000

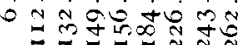
0 a r totentas क $\because 00000+00$

ठேீல்

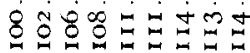

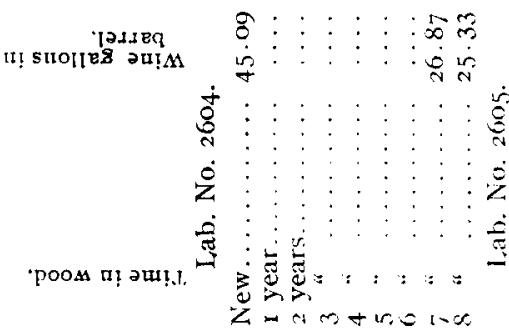

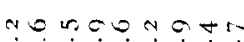

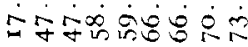
000000000

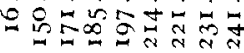

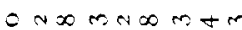
$\dot{0}-\dot{+} \dot{0} \dot{0} 0 \dot{0}$ o) pateinoter jolos

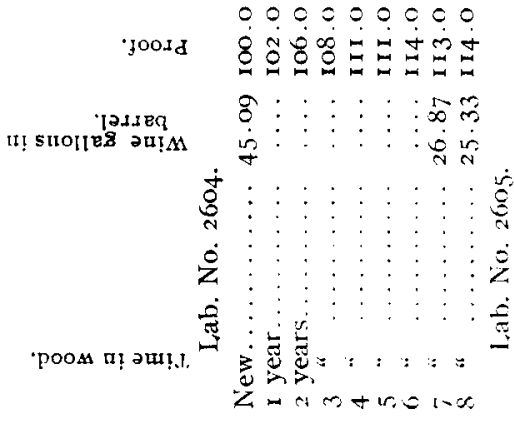

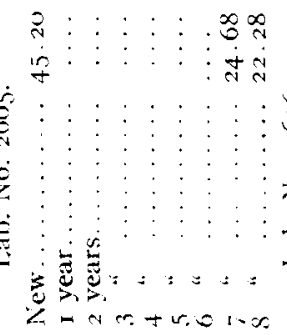
$\because 9-\infty \mathrm{n}+4 \mathrm{~N}$

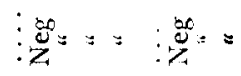

$\vdots \cos ^{\infty}=y:=:=$

. o. o. o. 00 $: \ddot{n}: \dot{n}: \dot{\sigma}: \dot{\sigma}$ o in 0000000 -

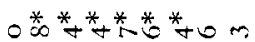

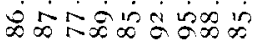

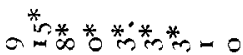

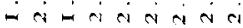

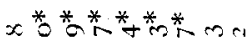

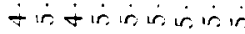

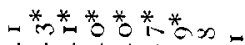

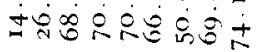

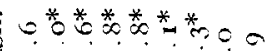

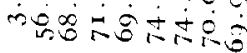
00000000

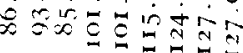
on 0 and and

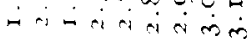

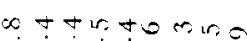

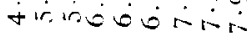
$=40000000$ करणि 0.0000000

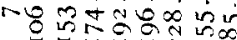
ormacomacos o 0000000

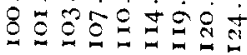
㧒: : : : : 8
8
8
-1 幽焉

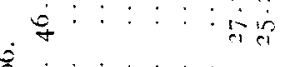

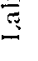




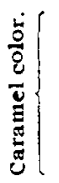

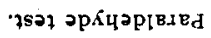

IวIEM

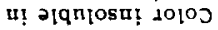

אq panouga jolo

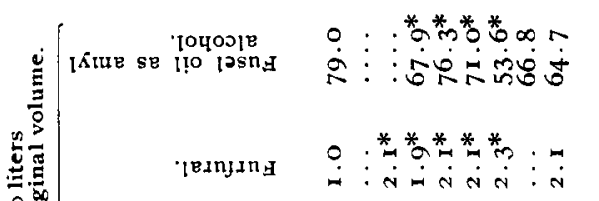

8 गпров se sats' INue se llo lasn

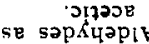

\section{0. * * * * * * .} $\dot{+}$ a $\dot{0} \dot{0} \dot{0}: \dot{1}$

$+: \sigma^{*}+* * * * 0$

- B̂600 in

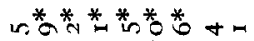
$\infty \infty \cdots \infty 0^{\infty} \infty \infty$ 。****** b $=\infty 0+0$, in: a o

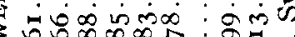

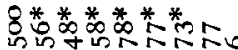

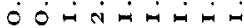

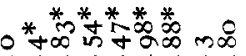

$\dot{\theta}+\infty \infty \infty \infty \infty$

$8 \pi$

ninoonmino

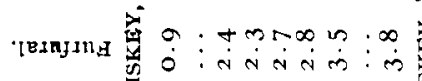

$\exists$

$a \cdot m+t \cdot \pi \cdot \pi$

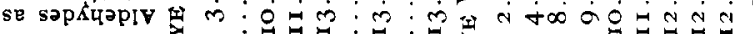
$\stackrel{4}{2}$

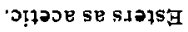

n.

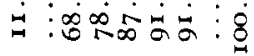

a. $n \pi \infty \infty 60$

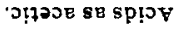

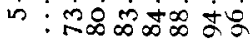

000000000

'spilos

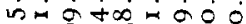

man

joond on on mantht

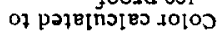

- $\dot{0} \dot{0} \infty \dot{0} \dot{0} \dot{0} \dot{0}$

000000000

jooxd

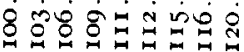

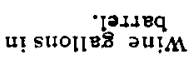

$\nexists: \vdots: \vdots:$ :

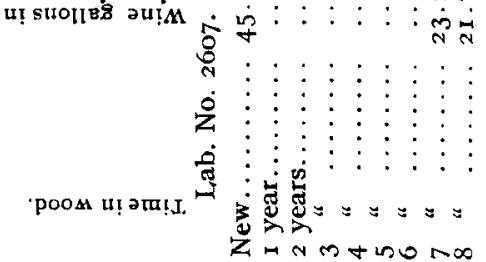

$n \infty \infty$ na nom

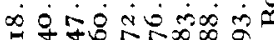

OMRenirron

- víñ

000000000

बूं मूँ

$0000 \pi$ m+ லம்

- $0000000 \mathrm{~N}$

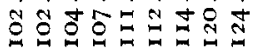
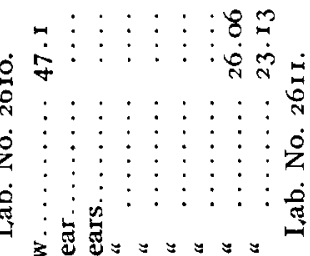

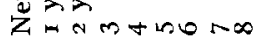

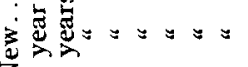

$: \mathbb{0}^{\infty}= \pm= \pm=:$

: 若 = = = =

$.0 .0: 0.00$

$: \infty: \sigma: \sigma: 5 \kappa$

nato00000

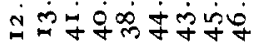

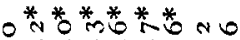

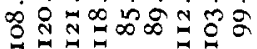

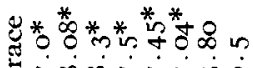
- $-\infty \infty \pi+n$

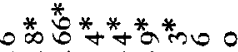

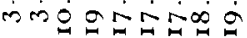

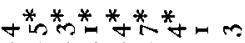
-

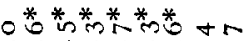

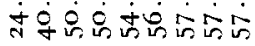
งด ดm

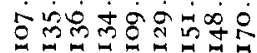
षam

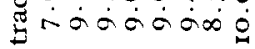

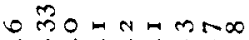
$\dot{m} \dot{\sim} \tilde{N}$ त $+\infty$ a m mNRo = オルna

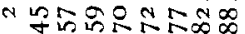
000000000

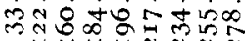
$\pi \pi m$ n ल N

唨 0 mm

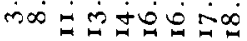
Mro 00 no $0 \mathrm{~m}$

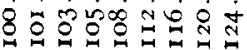
F: : : : : 유 is

क्ले

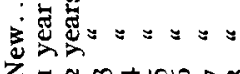




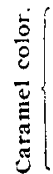

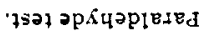

Iวjess

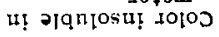

\section{Aq pasomat joto}

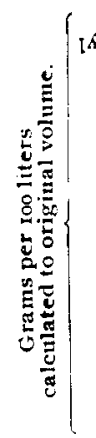

iste toyople Ito josna

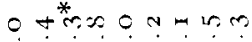
arinocon

jesmjatis

氖 20. SE $\operatorname{sap} \hat{\mathrm{T}} \mathrm{PIV}$ $\infty 0 \stackrel{*}{*}$ in $-0 \infty 0$. $-\infty * 00+00$

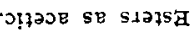

sाpכe se sptoy

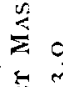

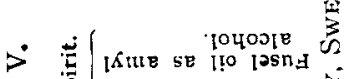
is

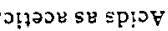

spifos

joord oor

$0 \propto 0 n+m, m$ of pazeinojes I0100

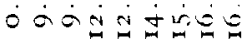
000000007 joord

\section{0}

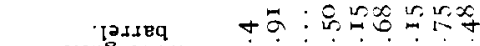
น! รนกำ วน!M i
$:$

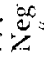

$0: 0: 0: 00$ $0 \infty 0000000$

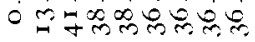

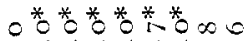

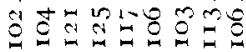

* ${ }^{*} \begin{gathered}* \\ 0\end{gathered}$ : 000 : $0 \dot{0} 0$

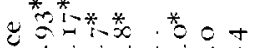

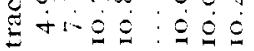

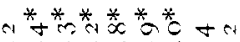

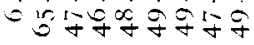

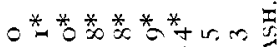

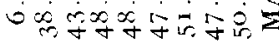
mo $00+3+100 \mathrm{~m}$

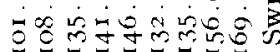

: FOR:POO - $0 \dot{0} 0$ : 000 $\int^{10}=-\infty \cdot n+\infty$

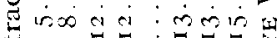

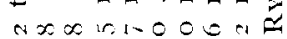
00 in in in is $0 x=m+t h-x$

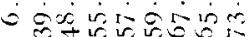
000000000 च o 采amanow

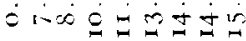
co 0000000

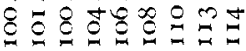

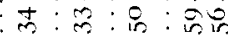

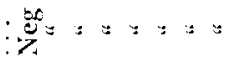

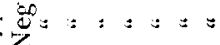
$0 \cdot 0 \cdot 0 \cdot 00$ in: $: \delta: \Omega$

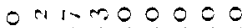
09

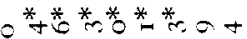

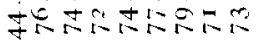

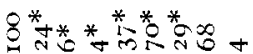

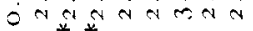

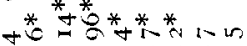

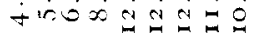

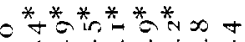
ज命

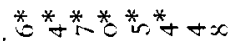

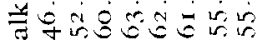

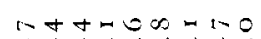

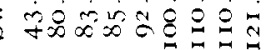

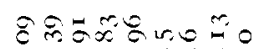

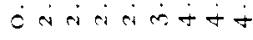
Foon wo on

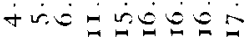

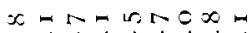

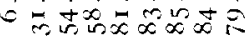
$\infty 0 m 0 x+m o$

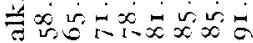
00000000

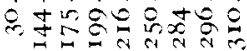

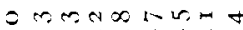
O OONOOOON

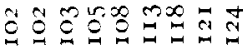

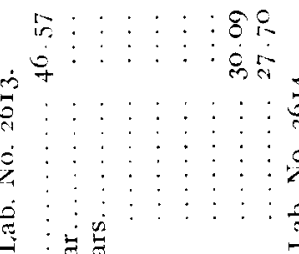

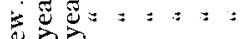

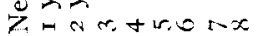

$\underset{2}{2}$ से त

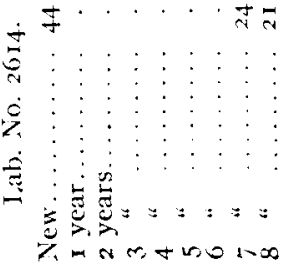



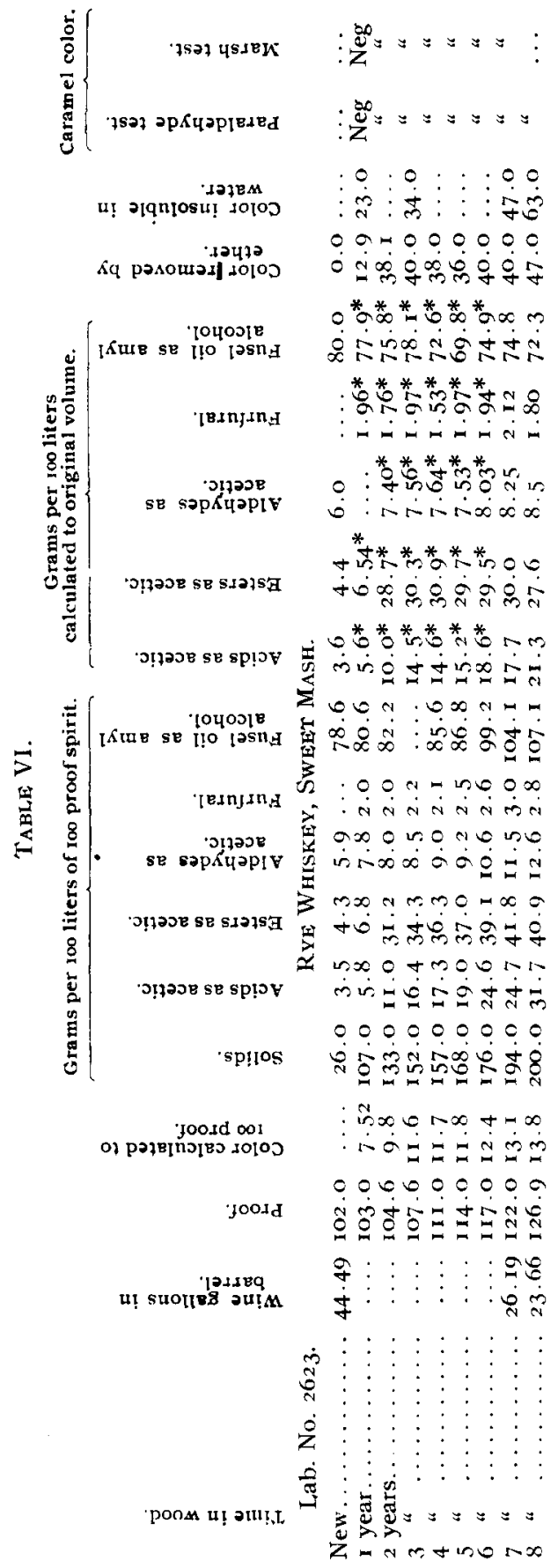


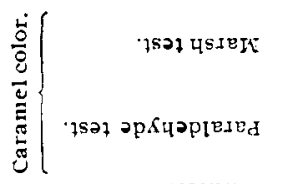

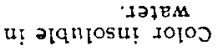

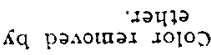

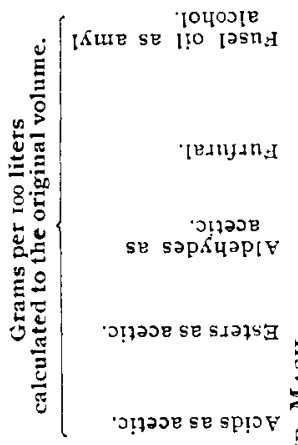

5
$: \frac{8}{20}=\div: \div:$

:

$\cdot 0 \cdot 0 \cdot 0 \cdot 00$

$: 0: 8: 5: 50$ o monolool

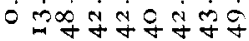
○满

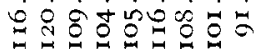

$8 \stackrel{*}{*} *$

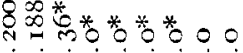

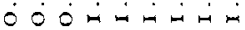

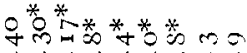

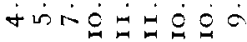

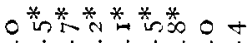

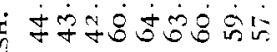

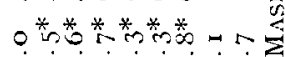

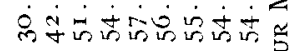
$800 \%$ mosoto IKue se lio lasnd

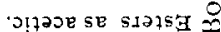
त

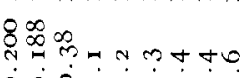

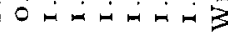

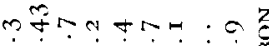

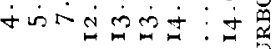
nagarat?

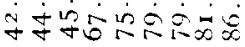
号

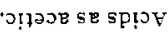
HNThNomat

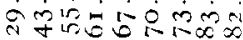
noo 000000

'splıos

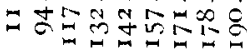

joond oOI of pajejnoteว noloj : ?90 nox 1. 000000000 ம்

jooid
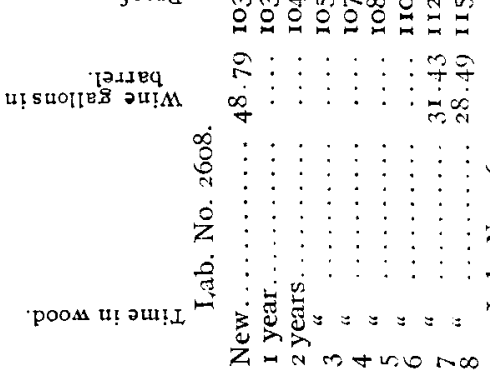

$: \mathscr{C O}^{\circ}:=:: \frac{80}{Z}$

$: \overbrace{0}^{\infty}::::=:=$

$0: 0: 0: 00$

m: n: in:

$0-\infty 000000$

0

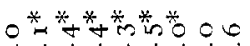

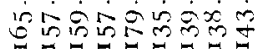

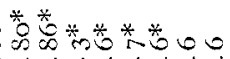

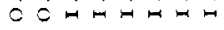

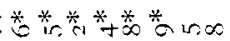

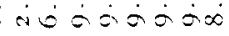

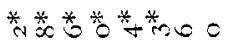
它它手安守守安

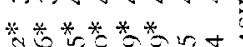
य $+\infty$ व $\dot{0} \dot{0}$ म

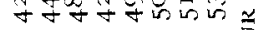

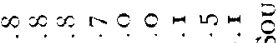
- 0 ormoniós

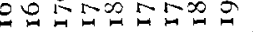
$8 \infty 00$ का

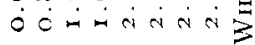
N8 un onnm

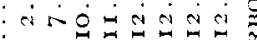
- ho osorolo

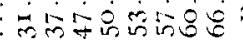
. $1000000 \mathrm{~mm}$

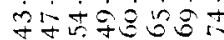
000000000

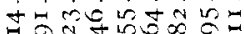

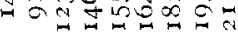

$\pi+t 0 m \infty$ r

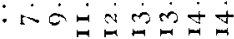
co0000000

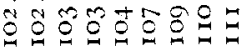

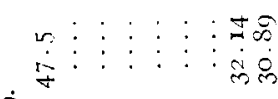

3 造

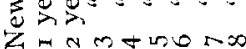

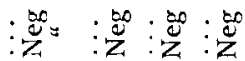

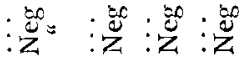

:0:0:0:00

: m: n: in: in

o rantoooco

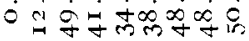

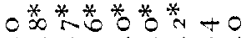

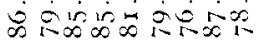

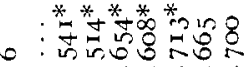

- 0000000

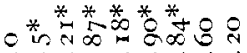

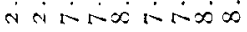

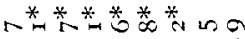

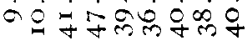

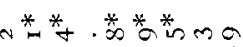

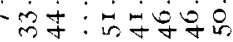
00000000

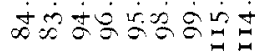

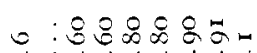
c:0 $000 \dot{0} 0$ $0+\infty \infty$ nom mo 0 तम r $+\infty$ an Mal a o $\mathrm{d}$ in $0=-9 \mathrm{~m}$

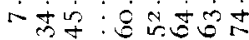
o 00000000 vob

a $0.0 \infty 0 \infty m$

- मr. : i ONOOOO nOO

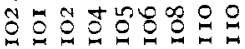

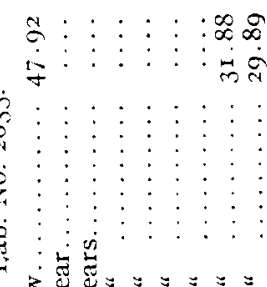

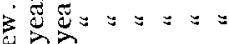

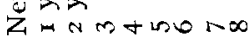




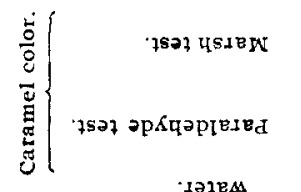

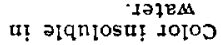

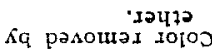

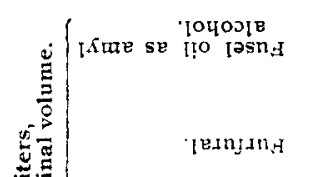

8

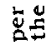

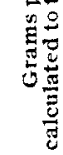

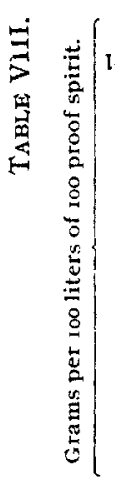

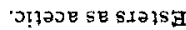

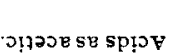

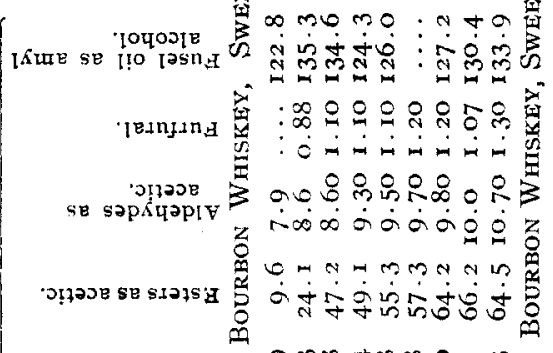

ว!ฺวอ se sp!of $a m \infty+\infty \infty \mathrm{OH}$ में 000000000

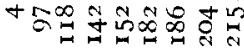

spilos

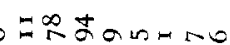
o) pajejnoter nojos

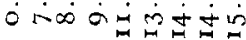
- 0 no o no o

joord

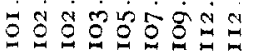

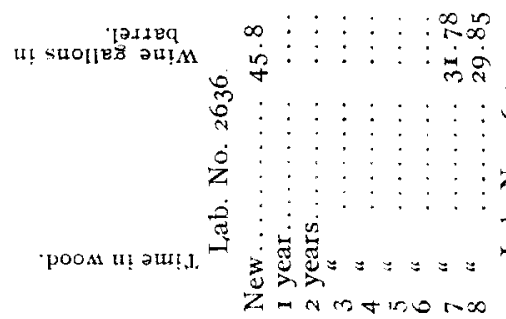

$: \frac{\infty}{2}=\square= \pm=$

$: \frac{\infty}{2}=: 4=:=$

.0 .0 .0 .00

: $8: 0$ :

o - no 00000

○节节节苍。0

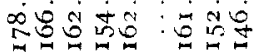

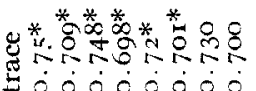

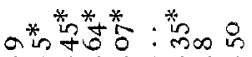
$\dot{0} \therefore-\infty \infty: \dot{\infty} \infty \dot{\infty}$

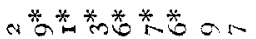

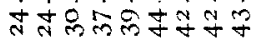

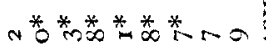

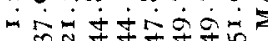
mपnoo.m a

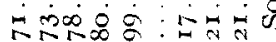
mIm $=$ तथ प요영ㅁ Ij $\dot{0} 0 \dot{0} \dot{0} \dot{0} \dot{0} \mathrm{~mm}$ $\operatorname{ann} 0 \mathrm{mam}$ on $\dot{0} \dot{0} 0$ : में o o o o oninat

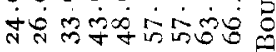

g 000000000

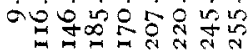
0 ल

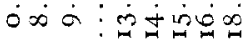
o nanocoloo

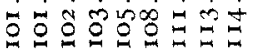
ㅇํㅇ : : : : : $\therefore \dot{\nabla}::: \infty_{N}$ \begin{tabular}{l}
5 \\
0 \\
0 \\
0 \\
\hline
\end{tabular} $\therefore \underbrace{2}$

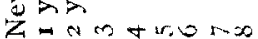

$::_{Z}^{\infty}=\square=\div=$

$: \mathbb{C}^{\infty}:=:=: 2$

$\cdot 0: 0 \cdot 0 \cdot 0=$

: $7: 0: 0: 000$

0 पा 000000

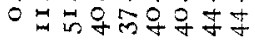

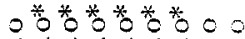

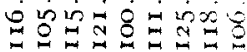

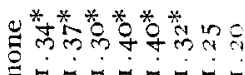

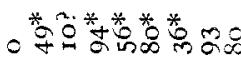

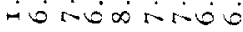

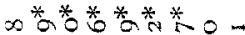

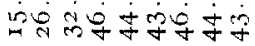

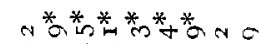

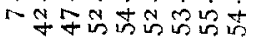

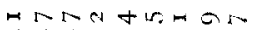
vion $200 \infty \infty$

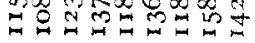

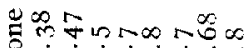
A $-m \rightarrow-m \rightarrow-$

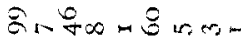

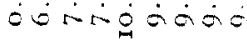

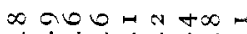
जू ले ar w 000 mo 000000000

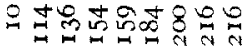

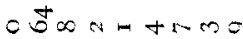
का NNOOOOOOU

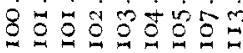
है: : : : : $:$ \%

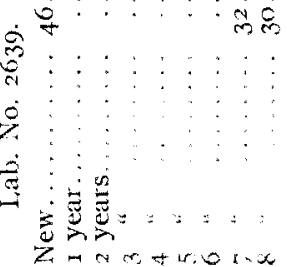




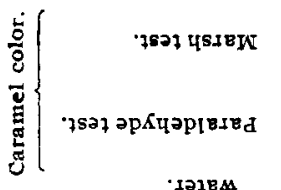

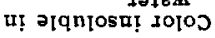

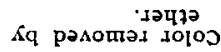
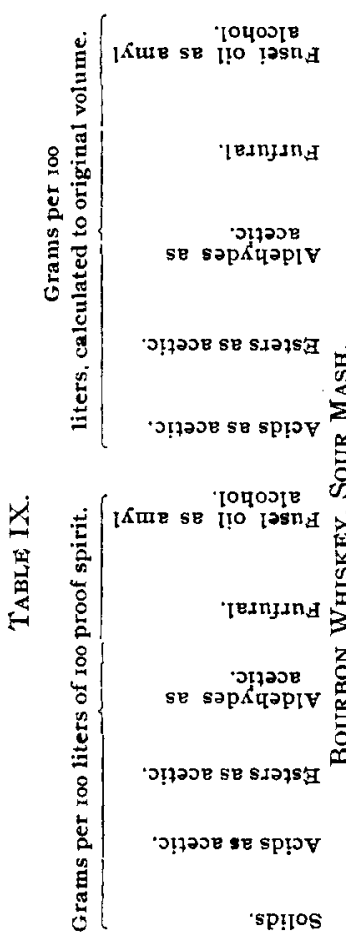

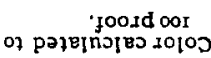

goodd

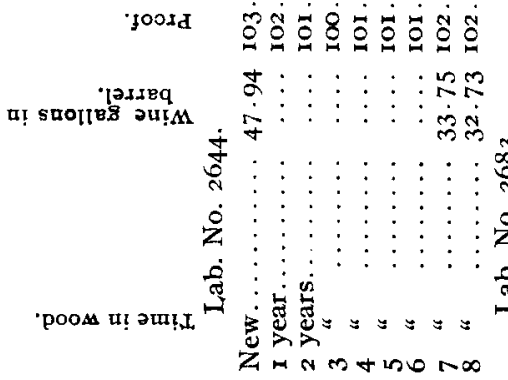

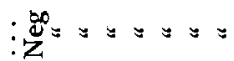

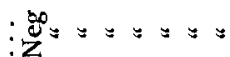

.000 .0 .00

$: 0 \ddot{0}: 00: \dot{0}$

on no 00000

ம்

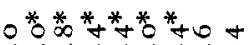

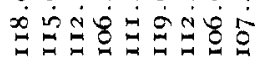

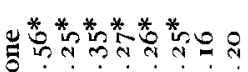

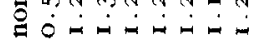

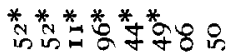

तरrionion

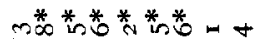

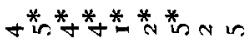

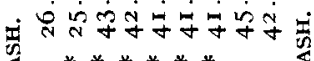
$\infty * * * * * * * * *$

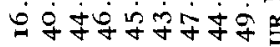

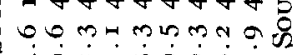

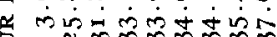

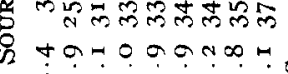

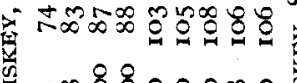
$: \infty 8 \& 8$ 요 $80 \%$ 空

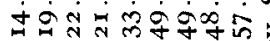

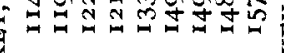
응요용요

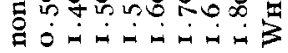
mos 800 mono तथं

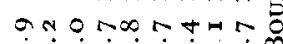

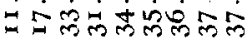
Andt+ho लं 800000000

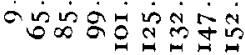

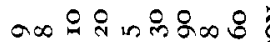
in் $\dot{0} \dot{\sigma} \dot{\sigma} \dot{0}$ o nom mo ox us ๙ mosoloo 00

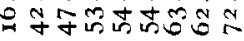
000000000 in $\infty$ i 0 i 0 - $\dot{+} \dot{0}-\infty \dot{0} \dot{0}$ nu $000000 \mathrm{~N}$

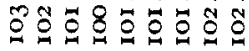

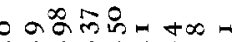

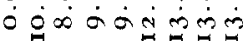
000000600

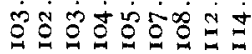

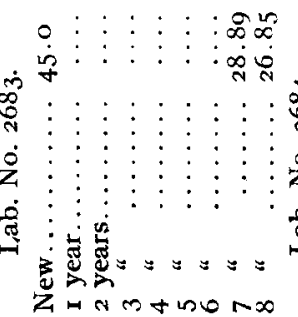

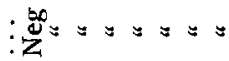

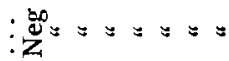

$: 0: 0: 0: 00$

: 000000000 的

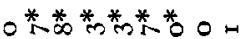

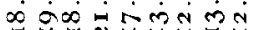

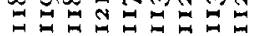

. ********o ज必乐合 $\dot{0} \dot{0} \dot{0} \dot{0} 0 \dot{0} 0$

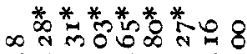

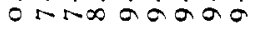

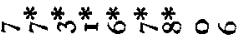
in

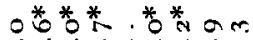
กำ $\infty$ n -

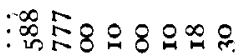
它亩向向向向

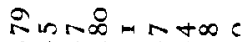

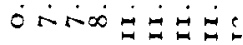
n acos ran ம் $a \infty 0 \infty$ : n $n \infty 0$ " a 0000000

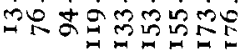
$0+$ ก๊ํำ 000 - $000 x$

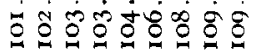
员: : : : : : น F $: \vdots: \vdots: \dot{m}$

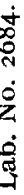

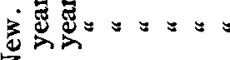
ZN $m+$ N $n$ No 


\section{它}

iales :0:0:0:00

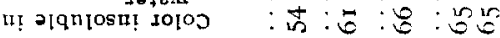

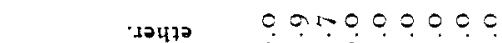
Kq prisurat

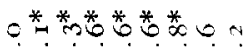

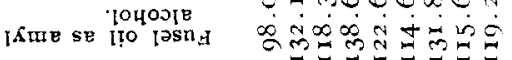
要

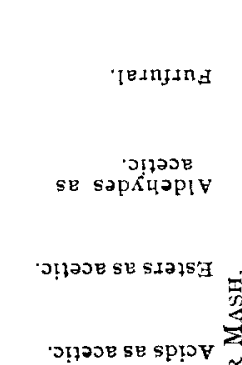

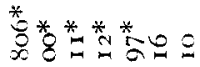
on $\dot{0} \dot{0} \dot{0}$

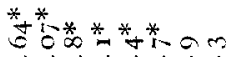
ता

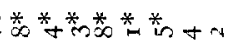

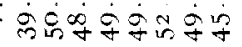

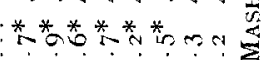

当

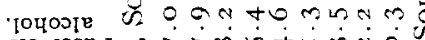

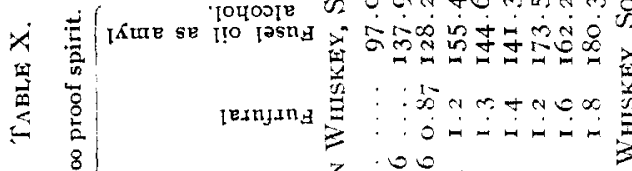

8
0
4
0
5
$\vdots$
8
8
5
4
0
0
$\vdots$
5
0 : De menemen

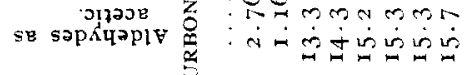
गाjave se siajs

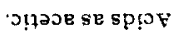

minenriog a n

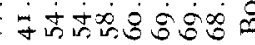
-

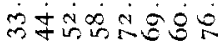
000000000 spllos क a) pajejiond oor

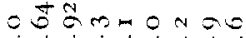
- 1ono num no

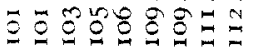

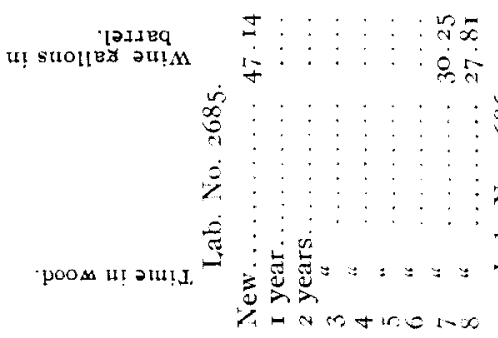

$: \stackrel{\infty}{*}=$

$: \otimes_{2}^{\infty}$

$: 0: 0: 0: 0=$ $: 4: 8: 5: 20$ gunocologo 0

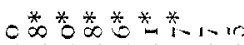
$\infty$ क क व 2000

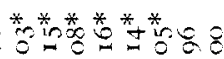
$\therefore \dot{m-i}-\dot{m}$

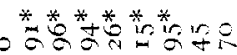
trive 0 os

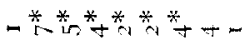

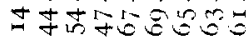

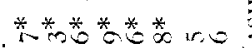

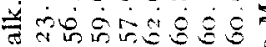
ง? $\pi \div+\because+\infty$

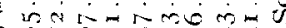

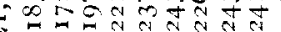
: N I $n \rightarrow-\dot{m}-\dot{m}$ ar. $\therefore \infty \infty \dot{0}=\dot{z}=$ Notela

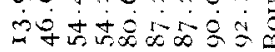
ㄴ? $000+4$ च 000000000

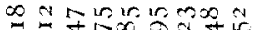
obramantwox - 0 a disis in 0 r. wh 000 m.

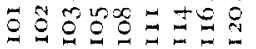

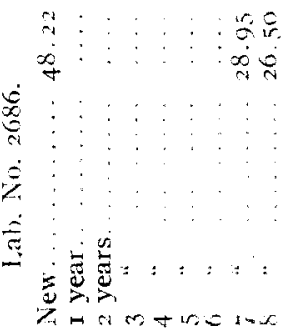

co:0.0 $+2: 00$ 00000000 की $0 \stackrel{0}{0}=n^{*}+\frac{*}{0} 0 \%$

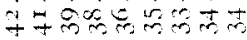

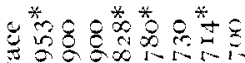
$=00030 \div 0$

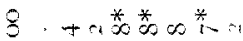

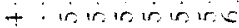

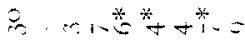
$-\therefore=0 \Rightarrow m$ or

$3+-\pi^{*}+\pi * a^{*}=$

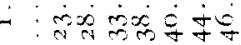
$0 x$ or w r -000

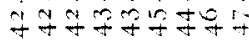

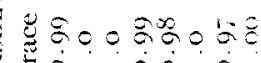

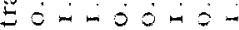
$0: 00001-1$. + iriserion $\Leftrightarrow \cdot a-\infty r^{2}, \ldots$

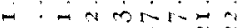

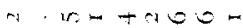
- ireconge 000000000

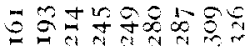

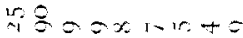
m 000000000

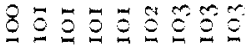

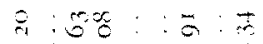

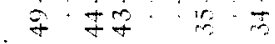
3

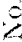

$\stackrel{5}{\vdots}$ $\div 8$ 

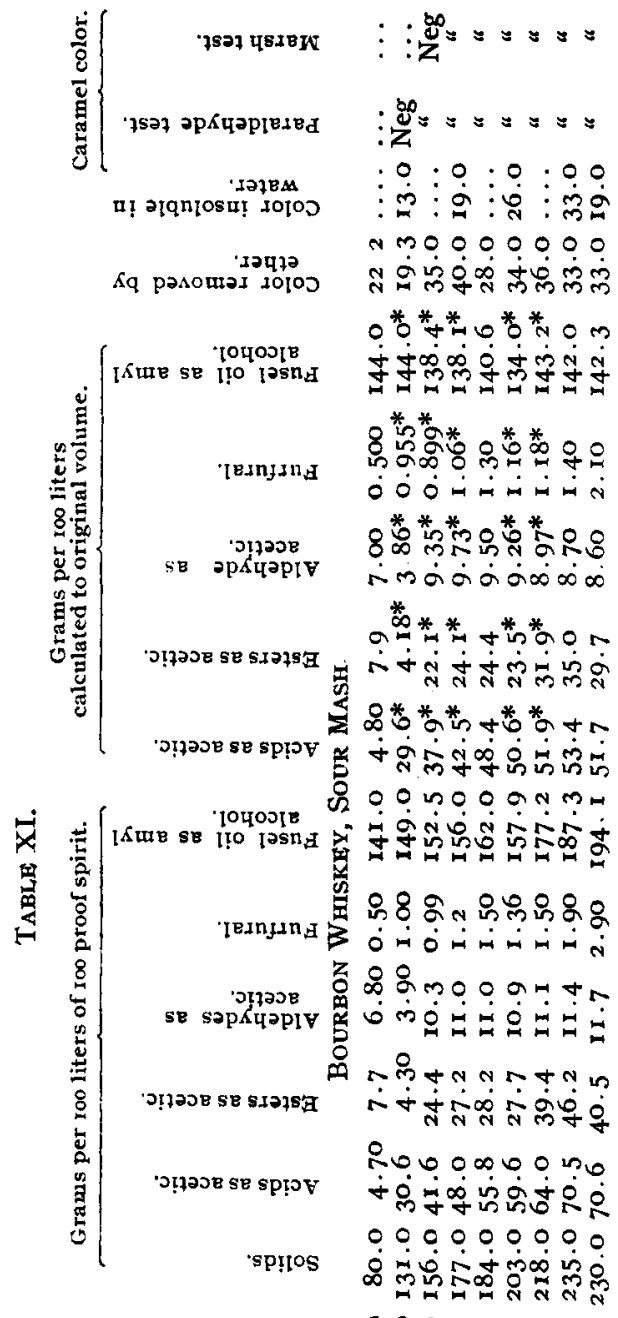

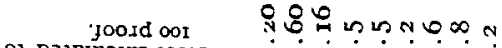

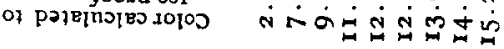
$000000 \pi 00$

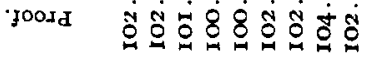

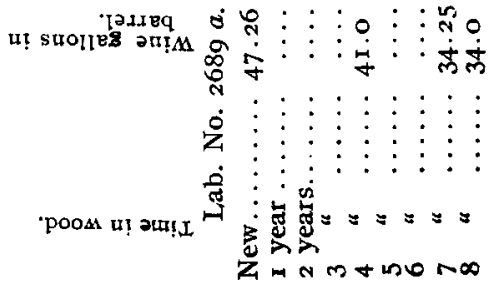




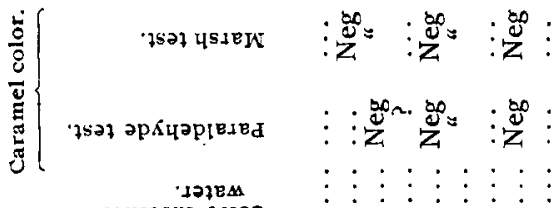
II! गाqniosu! JUIOS

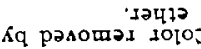

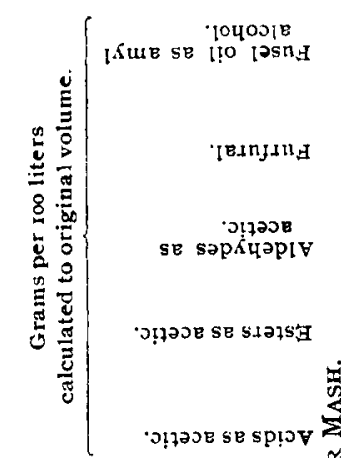

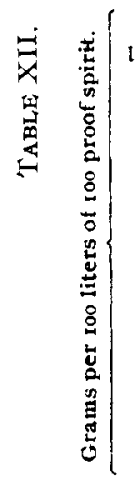

- jouosis

号

$0+n 000000$ -

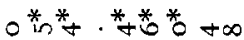

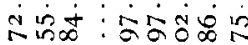

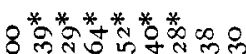

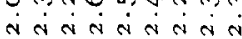

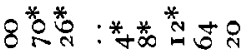
$\dot{m \infty} \infty \dot{\infty} \infty \dot{\infty} \infty$

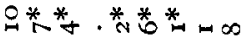
๑ 苾******

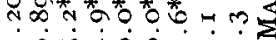
moO:OLnON

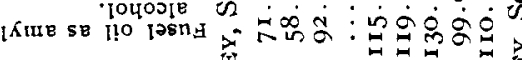

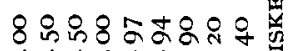

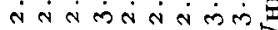

888.000

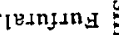
西 se 㟒

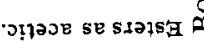
ㄴ. $\dot{0} \dot{0}: \because \div \%$

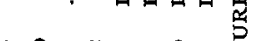
$\dot{m} \dot{4}: \dot{0} \dot{0} 0 \dot{0}$ nf: isำ

คู

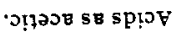
000000000

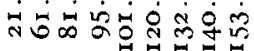

'sp!jos

joojd 001 o) pojelnoteo noloj

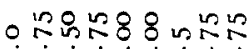
$\dot{0} \dot{\alpha} \dot{\pi} \dot{0} \dot{0} 0$ 000000000

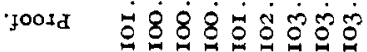

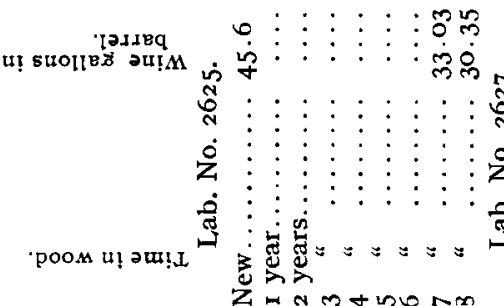

$\therefore ., i .0 \cdot 00$ - mmulo 000

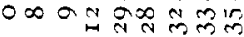

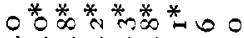

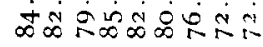

* $\stackrel{*}{*} *{ }^{*} * *$

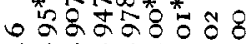
o 0 O 00 imm

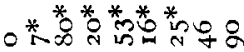

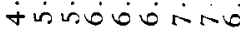

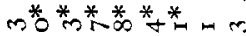

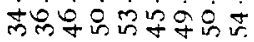

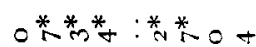
in non nmona $\dot{0} \cdot \dot{0} \cdot \dot{0} \cdot \dot{0}$

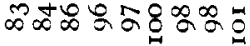

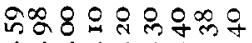

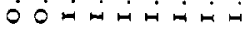
9aㅇㅇㅇㅇㅛ múrina ONONON+RH लिं aนnน 900000000

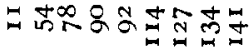

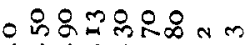

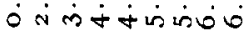
0 00000000

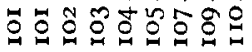

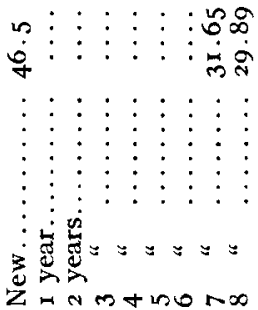




\section{Whiskey Aged in Uncharred Packages.}

No, 2627 (see T'able XII): Sour mash, Bourbon whiskey, entered into bonded warehouse February 5,1898 , in a new, uncharred, white oak, seasoned barrel (4 years old); warehouse, dry and above ground; average temperature in the winter $42^{\circ}$, in the summer $80^{\circ}$. Composition of the mash: malt, $6,048^{\circ}$ pounds; rye, 4,704 pounds; corn, 45,024 pounds. The spirit was distilled in a copper still of 5,182 gallons capacity, connected with a second still of 2,133 gallons capacity, and with a doubler of a capacity of 950 gallons. The spirit is taken directly from the doubler to the cisternroom without rectification or filtration. Yield per bushel of grain, 4 gallons. The flavor is rank, even in eight-year old goods, and very different from that of all samples aged in charred wood.

No. 2625 (see Table XII): Sour mash, Bourbon whiskey, entered into bonded warehouse January $3 \mathrm{I}, 1898$, in a new, uncharred, white oak barrel; low-boxed warelouse, about three feet from the ground; average temperature in winter $48^{\circ}$, in the summer $80^{\circ}$. Composition of the mash: malt, 9.52 pounds; rye, 448 pounds; corn, $s, 400$ pounds. Distilled from a column still, capacity 150 gallons per hour. No leach tubes used. Yield, 4 gallons of proof spirit per bushel of grain. Flavor not so rank as $\lambda(10.2627$. Odor, not like any American whiskey.

'iable Xill-Averace, Maxima and Minima Data on All the Samples of WHISKEY.

\begin{tabular}{|c|c|c|c|c|c|c|c|c|c|}
\hline $1 ;$ & $\begin{array}{l}\text { Data for } \\
\text { 3I samples. }\end{array}$ & Proof. & Color. & solids. & Acids. & Esters. & $\begin{array}{l}\text { Alde- } \\
\text { hydes. }\end{array}$ & Furfural. & $\begin{array}{c}\text { Fusel } \\
\text { oil. }\end{array}$ \\
\hline \multirow[t]{3}{*}{ New } & Average & IOL. & $\ldots$ & 20.1 & 6.4 & .3 & 3.9 & 0.9 & $95 \cdot 2$ \\
\hline & & 104.0 & & 161.0 & $20 \cdot I$ & & 15.0 & 2.0 & 171.3 \\
\hline & Min & 00.0 & $\ldots$ & 5.0 & 1. & & trace & race & 42.0 \\
\hline \multirow[t]{3}{*}{1 yr. } & Ave & 2.0 & 8.2 & IOO) 4 & 4.3 .6 & 6 & 6.7 & 1.7 & IIO 7 \\
\hline & & 4.0 & 1.3 .8 & 193. & 60 & .8 & 15.5 & & 194.0 \\
\hline & $\mathrm{Mi}$ & 00.0 & 4.6 & 54.0 & 5.8 & 6.8 & - & o. & 42.8 \\
\hline \multirow[t]{3}{*}{2 yrs. } & Ave & 3.6 & 10.1 & I 35. & 48.6 & 46.6 & $9 \cdot 3$ & & I 14,0 \\
\hline & & 100.0 & 16.7 & 214. & 63.8 &. \pm & Is. 7 & & 214.0 \\
\hline & Mir & 100.0 & 5.7 & 78.0 & II.O & .2 & .9 & & 42.8 \\
\hline \multirow[t]{3}{*}{3 yrs. } & Ave & $5 \cdot 2$ & 11.7 & $I 60.1$ & 58 & 8 & 1.9 & & 121.2 \\
\hline & & 112.0 & 18.3 & 245. & 81.8 & 9 & $22 . I$ & 9 & 202.0 \\
\hline & & & 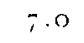 & & 16.4 & & & & 43.5 \\
\hline \multirow[t]{3}{*}{4 yrs. } & $A$ & 07.6 & 4 & 267. & 62.2 & $6 \mathrm{I} \cdot \mathrm{I}$ & t & & $125 \mathrm{k}$ \\
\hline & & I 18.0 & 18.9 & 249.0 & 83. & 89.1 & 22.2 & 9. & 237.1 \\
\hline & Min & 100.0 & $7 \cdot 4$ & 02.0 & $17 \cdot 3$ & 13.8 & 6.4 & 0.7 & $43 \cdot .7$ \\
\hline \multirow[t]{3}{*}{5 yrs. } & Aver & $09 \cdot R^{\prime}$ & $14 \cdot 1$ & 189.0 & 64.6 & 65.0 & 1.3 .1 & 2.5 & 126.8 \\
\hline & & 25.0 & 19.2 & 280.0 & 92.6 & $10.5 \cdot 5$ & 2.3 .1 & 9.6 & $254 \cdot 2$ \\
\hline & $\mathrm{Min}$ & IOI.O & 8.4 & II 4.0 & 19.0 & 17,3 & 6.6 & & 45.1 \\
\hline \multirow[t]{3}{*}{6 yrs. } & & 12.8 & 15.0 & 203 & 69.7 & 60.5 & 13.1 & & 139.9 \\
\hline & & 132.0 & $2 \mathrm{I} \cdot 2$ & 287.0 & 96.8 & 109.0 & $23 \cdot 7$ & & $245 \cdot 3$ \\
\hline & & 102.0 & $9 \cdot 3$ & 1.32 .0 & $24 \cdot 3$ & $1-9$ & 7.5 & 0 & 44.6 \\
\hline \multirow[t]{3}{*}{7 yrs. } & Ave & $195 \cdot 3$ & 1.5 .4 & 220.9 & $74 \cdot 3$ & 73.0 & 13.8 & & 141.0 \\
\hline & & $1+1.0$ & 227 & 3000 & 100.0 & 114.9 & 26.7 & $\therefore$. & 264.5 \\
\hline & Win & 102.0 & 10.1 & 1340 & $24 \cdot 7$ & $2 \mathrm{I} \cdot 3$ & 7.5 & 0.8 & +6.6 \\
\hline \multirow[t]{3}{*}{$\therefore y+$. } & Av & II 7,0 & 16.3 & 231.6 & 79.4 & 76.6 & $14 \cdot 3$ & 2.7 & 148.8 \\
\hline & & 1.34 .0 & $24 \cdot 2$ & .326 .0 & 112.0 & 126.6 & 28.8 & 10.0 & 280.3 \\
\hline & Minimum & IO2.0 & 10.5 & I 41,0 & 31.7 & 22.1 & 7.9 & 0.8 & 47.6 \\
\hline
\end{tabular}




\section{Discussion of Table XIII.}

This table shows the average, maxima, and minima data for each year on all of the thirty-one samples, with the exception that the fusel oil results on Sample No. 26I2, which was practically cologne spirits and the results obtained on the color and solids of samples Nos. 2625 and 2627 , which were aged in uncharred packages, were omitted.

The maximum and minimum figures alone have little value except to show the range obtained, because they do not establish any relationship between the various substances, as is done by the average figures.

One would not be justified in using these maxinum and minimum limits in judging the purity of the whiskey, because the results show that in a properly matured spirit the maximum in color and solids, for instance, does not occur with a minimum of acids and esters, so that if a sample of commercial whiskey should show a maximum color, it should not show the minimum acids and esters, and if such a condition were found, it would indicate that the product was a compounded article.

In the discussion of the analysis of a whiskey, all of the determinations and their relations must be considered before making a decision as to its purity. The simple fact that it falls between the limits shown by the maxima and minima figures is alone no definite indication as to its genuineness.

An interesting study might be made of the conditions which brought about these extreme results, which would be of practical value to the industry, but such discussion is beyond the scope of this paper. The facts alone will be presented, so that any one who is interested may interpret them.

It is evident from this work that there are two sources of furfural in whiskey. This is shown by the fact that some of the new distillates contain mere traces, while the mature spirits from the same source have considerable amounts, indicating that it must have been derived from the barrel; again, other samples of the fresh distillate contain considerable amounts, indicating that it must have come over in distillation and been derived from the grain of the mash. All of the samples, however, when calculated to the original (see Table 15 ) showed a slight increase of furfural on aging, which was undoubtedly derived from the charred wood.

\section{Discussion of Table XIV.}

This table gives the average, maximum and minimum of the determinations made on the rye whiskies.

The maxima, except on the color and solids, are of little value in judging the purity of other whiskies. These maxima for the various years give us limits for color and solids which will rarely be exceeded, and as shown by the average will be, as a rule, much less. The close relation- 
ship which should obtain between solids and color is shown by this chart. the sample showing the maximum color in each year also contains the maximum solids, except in the sixth year, where there is another santple showing slightly higher solids.

Table XiV.-Average, Maxima and Minima Data on Rye Whiskies.

Calculated to roo proof.

\begin{tabular}{|c|c|c|c|c|c|c|c|c|c|}
\hline \multirow{4}{*}{$\begin{array}{l}\text { Age. } \\
\text { New }\end{array}$} & Wata. & $\begin{array}{c}\text { Originsl } \\
\text { ptoof. }\end{array}$ & Color. & Solids. & Acids. & Fisters. & $\begin{array}{l}\text { Alde. } \\
\text { hydies. }\end{array}$ & Furfural. & $\begin{array}{l}\text { Fuse! } \\
\text { oil. }\end{array}$ \\
\hline & Average..... & 101.2 & 0.0 & $13 \cdot \hat{3}$ & $4 \cdot 4$ & 16.3 & $5 \cdot 4$ & I. 0 & 90.4 \\
\hline & Maximutu , . & 102.0 & 0.0 & 30.0 & 72.0 & 21.8 & 15.0 & 1.9 & I6I. 8 \\
\hline & Minimum.... & 100.0 & 0.0 & 5.0 & I 2.0 & $4 \cdot 3$ & 0.7 & trace & $\left\{\begin{array}{l}01.8 \\
43.7\end{array}\right.$ \\
\hline \multirow[t]{4}{*}{ I $y r$. } & Average.... & 102.5 & 8.8 & 119.7 & 46. & 37. & 7.0 & I. 8 & $\mathrm{III.5}$ \\
\hline & Maximum ... & 104.0 & 13.8 & 171.0 & 60.5 & 64.8 & 15.5 & $3 \cdot 3$ & 194.0 \\
\hline & Mininumm & IOI 0 & $7 \cdot 2$ & 93.0 & $33 \cdot I$ & $6.8\}$ & 28 & 0 & \\
\hline & 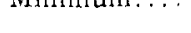 & 101.0 & & 92.0 & & & 2.0 & 0.4 & 66.4 \\
\hline \multirow[t]{4}{*}{2 yrs. } & Average & 104.9 & I 1.6 & 144.7 & 51.9 & 54.0 & 10.5 & 2.2 & $\operatorname{II} 2.4$ \\
\hline & Maximum & 109.0 & I 6.7 & 199.0 & 75.6 & 75.1 & 18.7 & $5 \cdot 7$ & 214.0 \\
\hline & & & 8.8 & 121.0 & $44 \cdot 3$ & $4 I .5\}$ & & & 83.4 \\
\hline & Mini & 100. & 18.6 & 94.0 & II.O & $3 I .25$ & $5 \cdot 4$ & 0.7 & 182.2 \\
\hline \multirow[t]{4}{*}{ yrs. } & Averag & 107.7 & 13.2 & 171.4 & 62.7 & $6 x \cdot 5$ & 12.5 & I. 5 & $\mathrm{r} 12.7$ \\
\hline & Maxim & 112.0 & IS. 3 & 224.0 & 81.8 & 79.8 & 20.8 & 6. I & 202.0 \\
\hline & Vin & 104. & II I.4 & 145.0 & $52 \cdot 3$ & $47.6\}$ & $.5+3$ & 0. & \\
\hline & 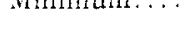 & 104.0 & (10.I & 119.0 & I 6.4 & $34 \cdot 35$ & $\cdot 5$ & 0.7 & 160.0 \\
\hline \multirow[t]{4}{*}{4 yrs. } & Avera & IIII. 2 & I 4.0 & 185.0 & 65.9 & $69 \cdot 3$ & $\mathrm{I}_{3} \cdot 9$ & 2.8 & 125.1 \\
\hline & Maximum ... & I 18.0 & I 8.9 & 2.38 .0 & 83.8 & 89.1 & 22.1 & 6.7 & $203 \cdot 5$ \\
\hline & Minimr & 105.0 & I I. 6 & 156.0 & $5^{8}$ & & 6,4 & $0.7 \quad-$ & \\
\hline & & & 211.3 & 153.0 & $17 \cdot 3$ & 36.35 & & & \\
\hline \multirow[t]{4}{*}{5 yrs. } & Average & 113.8 & I 5.9 & 206.5 & 67.6 & 75.0 & I 5.0 & 3.2 & $\mathrm{I} 28 . \mathrm{I}$ \\
\hline & Maxim & 125.0 & 19.2 & 251.0 & 92.6 & 105.5 & 22.4 & $7 \cdot 7$ & 254.2 \\
\hline & Minimum & 108.0 & & 170.0 & 0.4 & $60.5 ?$ & 6.6 & 1.4 & \\
\hline & & & (I) & & & & & & \\
\hline \multirow[t]{4}{*}{6 yrs. } & Averag & 118.0 & 17.0 & $223 \cdot I$ & 72.4 & 80.4 & 14.6 & $3 \cdot 3$ & $x 45 \cdot 5$ \\
\hline & $\operatorname{Max}$ & 132.0 & 21.2 & 284.0 & 95.8 & 109.0 & $22 \cdot 3$ & $8 \cdot 3$ & $245 \cdot 3$ \\
\hline & Minimun] & 110.0 & $\int 13.7$ & & 7 & $64.0\}$ & $7 \cdot 3=3$ & 0.7 & \\
\hline & & & $1 \mathrm{I} 2.4$ & & & $39-1)$ & & & 0.0 \\
\hline \multirow[t]{3}{*}{$7 \mathrm{yrs}}$. & Aver & 121.4 & Is. 0 & 242.2 & $7^{6} \cdot 7$ & $84 \cdot 2$ & $15 \cdot 5$ & $3 \cdot 2$ & $145 \cdot 2$ \\
\hline & Maximun & $\mathrm{I}_{4} \mathrm{I} . \mathrm{O}$ & 22.7 & 306.0 & 100.0 & I I 4 . & $25 \cdot 2$ & 8.5 & $264 \cdot 5$ \\
\hline & Minimum & III.O & $13 . I$ & 195.0 & 60.9 & & $7 \cdot 5$ & 0.8 & \\
\hline \multirow[t]{4}{*}{ yrs. } & Average. . & 123.8 & 18.6 & 256.0 & 82.9 & 89.1 & 16.0 & $3 \cdot 4$ & \\
\hline & Maximu & 132.0 & $24 \cdot 2$ & 339.0 & 112.0 & 126.6 & 26.5 & 9.2 & 280.3 \\
\hline & & I I 2.0 & $\{13.8$ & 214.0 & $73 \cdot 7$ & $68.4\}$ & & 0.8 & o \\
\hline & & & 213.7 & 200.0 & $3 \pi \cdot 7$ & 40.9 & $7 \cdot 9$ & Ho & $107 \cdot 1$ \\
\hline
\end{tabular}

The minina, however, are of considerable interest, not so much for color and solids as for the acids and esters. Taking the acids for instance, the minimum for each year is given by one package, No. 2623, which is abnormal in many ways, and omitting it from consideration the minimum 
of acids for the first year would be 33.I, only slightly below the average; for the second year it wopld be 44.3 , and so on.

In order to show this and to eliminate the abnormal, the next to the lowest figures for the color, solids, acids, esters, and fusel oils are also included in the table.

Table XIVa.-Average, Maxima and Minima Data for Bourbon Whiskies.

Calculated to roo proof.

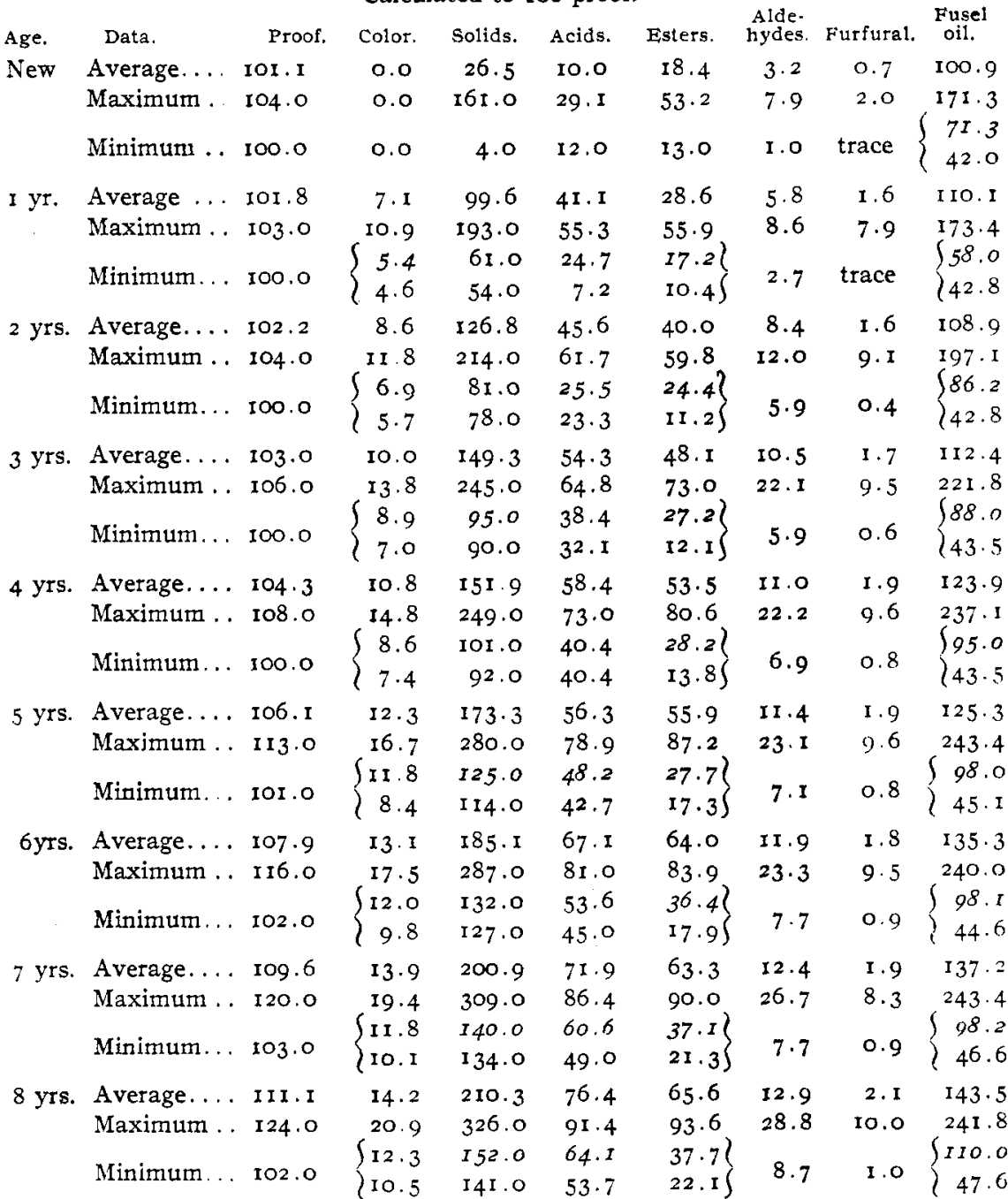

The point is especially noticable in the esters, for, omitting the minima, the lowest ester found on a four-year old whiskey was 57.7 grams, which is only slightly below the average, and would indicate that one might 
expect in a four-year old rye whiskey 50 to 60 grams of esters to 100 liters.

Table XIV shows the average, maxima and minima data for the Bourbon whiskies.

The same may be said of this table as of the previous one in regard to the value of the maxima and minima figures.

All of these results indicate that we should expect lower figures with Bourbon whiskies than with rye.

The high solids shown in the maximum for the new spirit was due largely to ash, this being sample 2689 , and the high ash changes, in this particular sample, the ratio between the color and solids.

TAble XV.-Ayerage Data for All Samples and for Rye and Bourbons SepaRATELY.

Calculated to original volume.

\begin{tabular}{|c|c|c|c|c|c|c|c|c|}
\hline \multirow{4}{*}{$\begin{array}{l}\text { Age. } \\
\text { New }\end{array}$} & original volume. & Color. & Solids. & Acids. & Fsters. & dehy & urfu & aselo \\
\hline & Whole......... & $\ldots$ & 20.0 & 6.4 & 15.0 & 4.03 & $0.7 \mathrm{I}$ & 96.8 \\
\hline & Rye.......... & $\ldots$ & 13.6 & 4.7 & $13 \cdot 7$ & 4.91 & 0.97 & 83.2 \\
\hline & Bourbon........ & $\ldots$ & 26.0 & 7.7 & 17.2 & 3.26 & 0.44 & 108.6 \\
\hline \multirow[t]{3}{*}{$1 \mathrm{yr}}$. & Whole......... & $7 \cdot 3$ & 101.5 & 37.8 & 29.9 & 7.08 & I. 5 & 106.2 \\
\hline & Rye. & 8.4 & 154.6 & 41.8 & $35 \cdot 3$ & 8.75 & I. 7 & 106.8 \\
\hline & Bourbon. . & 6.4 & 90.1 & $34 \cdot 4$ & 24.9 & $5 \cdot 55$ & 1.3 & 105.8 \\
\hline \multirow[t]{3}{*}{2 yrs. } & Whole... & 8.6 & $124 \cdot 2$ & 46.1 & 42.9 & 8.34 & r.: & 108.1 \\
\hline & Rye. & 10.6 & 133.6 & 49.8 & $49 \cdot 3$ & 9.02 & 1,9 & $109 \cdot 7$ \\
\hline & Bourbon. & 6.7 & I 14.8 & 42.7 & $37 \cdot 3$ & 7.78 & I, 4 & $107 \cdot 3$ \\
\hline \multirow[t]{3}{*}{3 yrs. } & Whole.... & 10.2 & 140.2 & 51.1 & 48.4 & $9 \cdot 4 i$ & I. 8 & $106 \cdot 3$ \\
\hline & $\ldots \ldots \ldots$ & 11.5 & 150.4 & $.54 \cdot 4$ & $54 \cdot 3$ & 9.80 & 2.2 & $104 \cdot 4$ \\
\hline & Bourbon........ & 8.3 & 130.7 & 47.8 & $42 \cdot 5$ & 9.15 & 1.5 & $107 \cdot 3$ \\
\hline \multirow[t]{3}{*}{4 yrs. } & Whole. & 10.2 & 140.4 & 51.6 & 50.9 & 10.2 & 1.9 & $104 \cdot 3$ \\
\hline & Rye & II. 6 & I 53,1 & 54.2 & $57 \cdot 2$ & II .2 & 2.2 & 102.0 \\
\hline & Bor & 8.9 & 127.7 & 48.9 & $45 \cdot 0$ & $9 \cdot 3$ & I. 5 & 106.3 \\
\hline \multirow[t]{3}{*}{5 yrs. } & Whole. & II. I & $149 \cdot 2$ & .52 .2 & $5 I . I$ & 10.2 & I.9 & 100.4 \\
\hline & Rye. & 12.2 & 158.8 & 54.8 & $57 \cdot 5$ & I I 3 & 2.5 & 100.1 \\
\hline & Bour & 10.0 & 140.2 & 49.8 & 45.0 & $9 \cdot 2$ & I. 5 & 100.7 \\
\hline \multirow[t]{3}{*}{6 yrs. } & Whole. & I I I I & $15 I .4$ & $.53 \cdot 2$ & 50.7 & 10.2 & I.9 & $104 \cdot 7$ \\
\hline & Rye. & $\mathrm{I} 2.3$ & $I 61.0$ & 54.8 & $55 \cdot 5$ & I I. 3 & $2 \cdot 4$ & $105 \cdot 9$ \\
\hline & Bourbon........ & IO. I & 142.5 & 51.8 & $45 \cdot 2$ & $9 . I$ & 1.4 & 103.8 \\
\hline \multirow[t]{3}{*}{7 yrs. } & Whole......... & II, I & 154.0 & $.52 \cdot 2$ & $51 \cdot 1$ & 9.8 & I. 8 & 99.9 \\
\hline & Rye. & 12.0 & $16 \mathrm{r} \cdot 3$ & 51.9 & 56.6 & 10.6 & 2.2 & 98.8 \\
\hline & Bourbon........ & 10.2 & $147 \cdot 1$ & $52 \cdot 4$ & 46.4 & 9.0 & 1.4 & 101.6 \\
\hline \multirow[t]{3}{*}{8 yrs. } & Whole......... & 10.5 & I 55.2 & $53 . I$ & 50.9 & 9.6 & 1.8 & 98.0 \\
\hline & Rye......... & I I. I & I63.8 & 52.6 & 56.7 & 10.6 & 2.2 & 99.0 \\
\hline & Bourbon........ & I0.0 & 147.7 & 53.6 & 45.9 & 8.8 & I. 5 & $97 \cdot 1$ \\
\hline
\end{tabular}

This table gives the average of all the whiskies and the average of the rye and Bourbon separately, calculated back to the original volume, in order to eliminate the effects due to the concentration taking place while the spirit was stored. The more important points brought out by this table are brought out in the various charts. These figures show 
more nearly the actual changes taking place, and prove that after the third year there is very little change in the amounts of the various substances present. As has been said before, the changes taking place in the whiskey after the third or fourth year are almost entirely due to the concentration which occurs.

These figures also show that the difference between rye and Bourbon whiskies holds good even when the results are corrected for loss of volume, proving that with the rye whiskies there has been a greater activity in the aging processes.

The fact that after the third year, the acids, esters, and aldehydes do not show any appreciable increase when corrected for loss in volume, does not necessarily mean that there is no formation of these substances in the barrel, as there may be some loss of them through the wooden walls of the barrel, with the alcohol and water which is constantly passing off; but the fact that they remain so constant in amount would indicate that these substances are left behind, in the same manner as the fusel oil and solids, by what might be called the selective action of the wooden membrane constituting the walls of the porous cell in which these products are stored.

Chart I shows the average of the changes taking place in the proof and the volume.

The proof increases, starting from approximately roo proof, so that all that is shown on the curve are the degrees above Ioo proof.

The changes in volume are plotted from the per cent. of loss in volume. The changes in proof and volume are important in their bearing on the changes that take place in the other substances.

The wide difference in rate of increase of proof shown by the rye and Bourbon whiskies is typical of the differences between them, and apparently has a simple explanation. In the case of the rye whiskies nearly all were aged in heated warehouses where the changes taking place are aided by the higher temperature. There is also a large loss of volume in the rye whiskies, as is shown by the lines. In short, there is much greater chemical activity, and so, greater changes. The amounts of acids and esters formed apparently have a direct relationship to the amount of the whiskey which passes through the wood of the barrel.

The Bourbon whiskies, on the other hand, are as a rule stored in unheated warehouses, so that there is less loss of volume in the spirit, and less increase in proof, and snualler amounts of acids and esters are formed. As a matter of fact, a much lighter bodied whiskey is formed, and from these results it would seem to be due to a greater extent to the method of storage than to the inherent differences in the whiskies themselves.

Chart II shows changes in solids and coloring matter, and the sane differences between the rye and Bourbon whiskies as were noted on Chart 


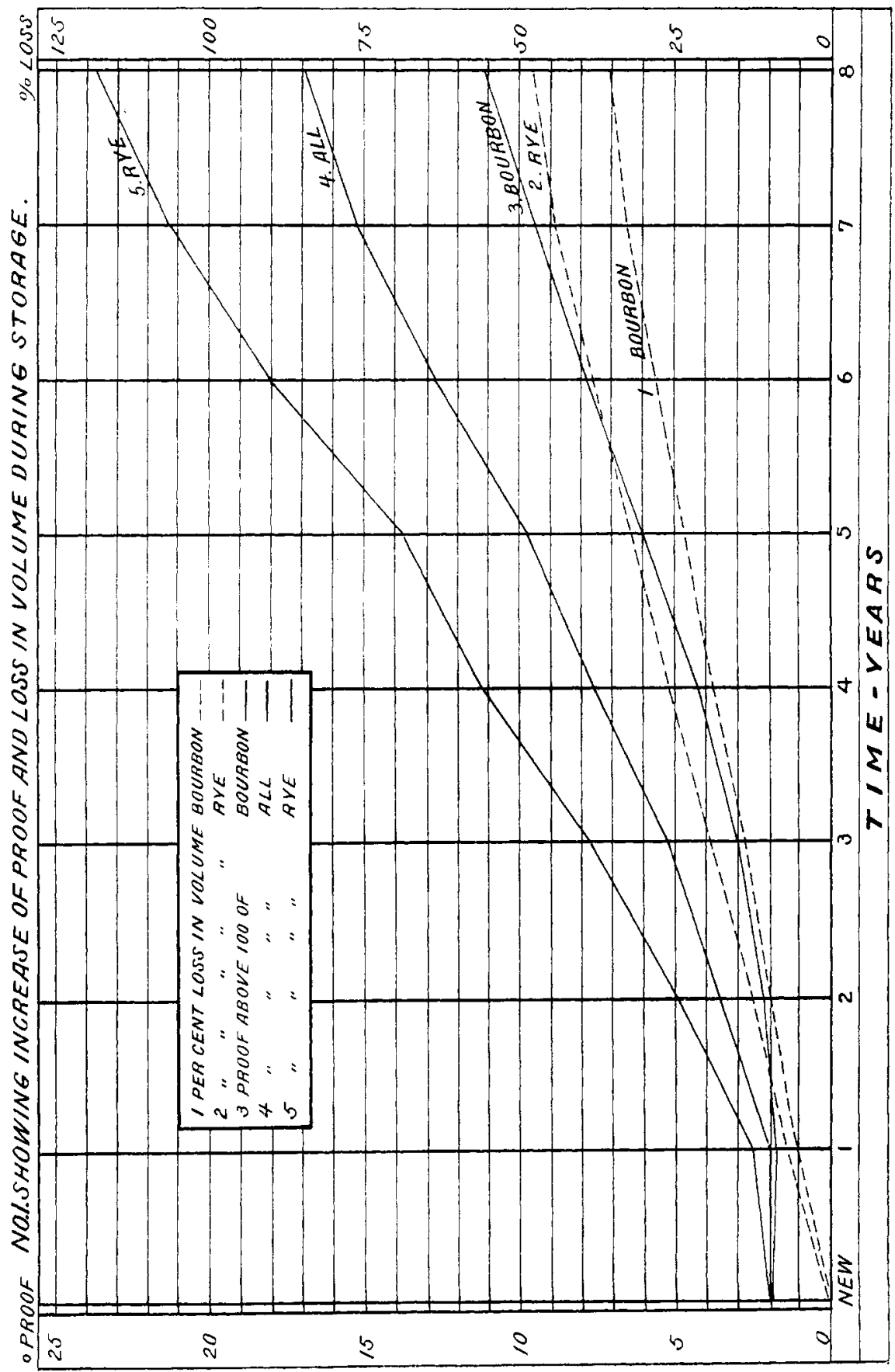




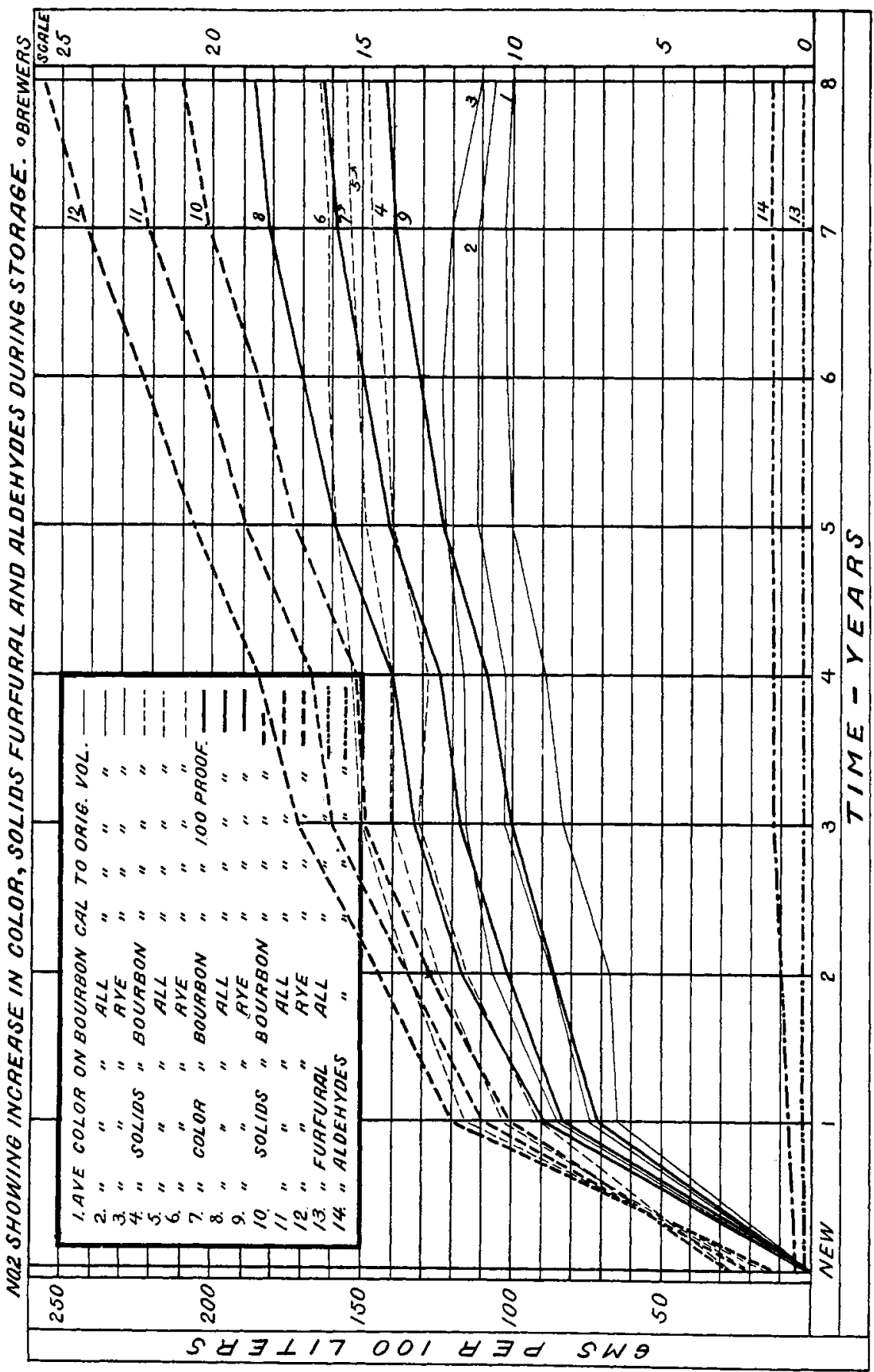


I are seen, namely, a marked increase during the first year, and a regular increase thereafter.

A comparison of the solids as calculated to 100 proof, and as calculated to the original volume, shows that the increase in the former is almost entirely due after the third year to the concentration taking place, the actual amount of solid matter in solution in any single barrel remaining practically the same after the third year. The same is true of the coloring matter and apparently little material is extracted from the wood after that time.

The remarkable similarity of the solids and color curves shows that there is a very close relationship between them. In fact, the relationship is so close that from the determination of the solids one could very closely calculate what the color should be, or from the color calculate the solids.

This relationship is of great value in the detection of the artificial coloring of whiskey, and in judging whether the solids are normal.

Chart III shows first the changes in fusel oil, calculated to 100 proof, and to the original volume. A gradual increase of fusel oil is shown as the whiskey ages, but it will be seen from the curve plotted from the calculations to the original volume that this change is due to concentration. The actual amount of fusel oil in a barrel of whiskey remains the same during the period of storage, but the whiskey itself shows a percentage increase.

These results, however, do not prove whether the fusel oils undergo change in aging, or whether any of the fragrant esters present are due to the fusel oils, because the method of analysis employed, first saponifies all of the esters and determines only the higher alcohols. The question as to the change taking place in the fusel oils must be answered by fractionating large quantities of a new and of a mature spirit and studying the composition of the higher boiling-point portions.

This chart also shows the changes occurring in the acids and esters, and further emphasizes the fact of the differences between rye and Bourbon whiskies. In every way the rye whiskies are the stronger, if one may use the term; that is, they contain more solids, color, acids, esters, etc., but all of these differences can be traced to the method of aging.

A very striking point which is also brought out by this chart is the change in relationship between the acids and esters in young spirits and the constant relation in matured spirits.

The average of the acids in the new spirit is 6 grams per Ioo liters, and the esters average 16 . In the spirit one year old this relation has changed, the acid being 44 grams against 33 grams for the esters. The second year the acids are 49 , and the esters 47 . The third year the acids are 59 , and the esters 5.5 . The fourth year the acids are 62 and the es- 


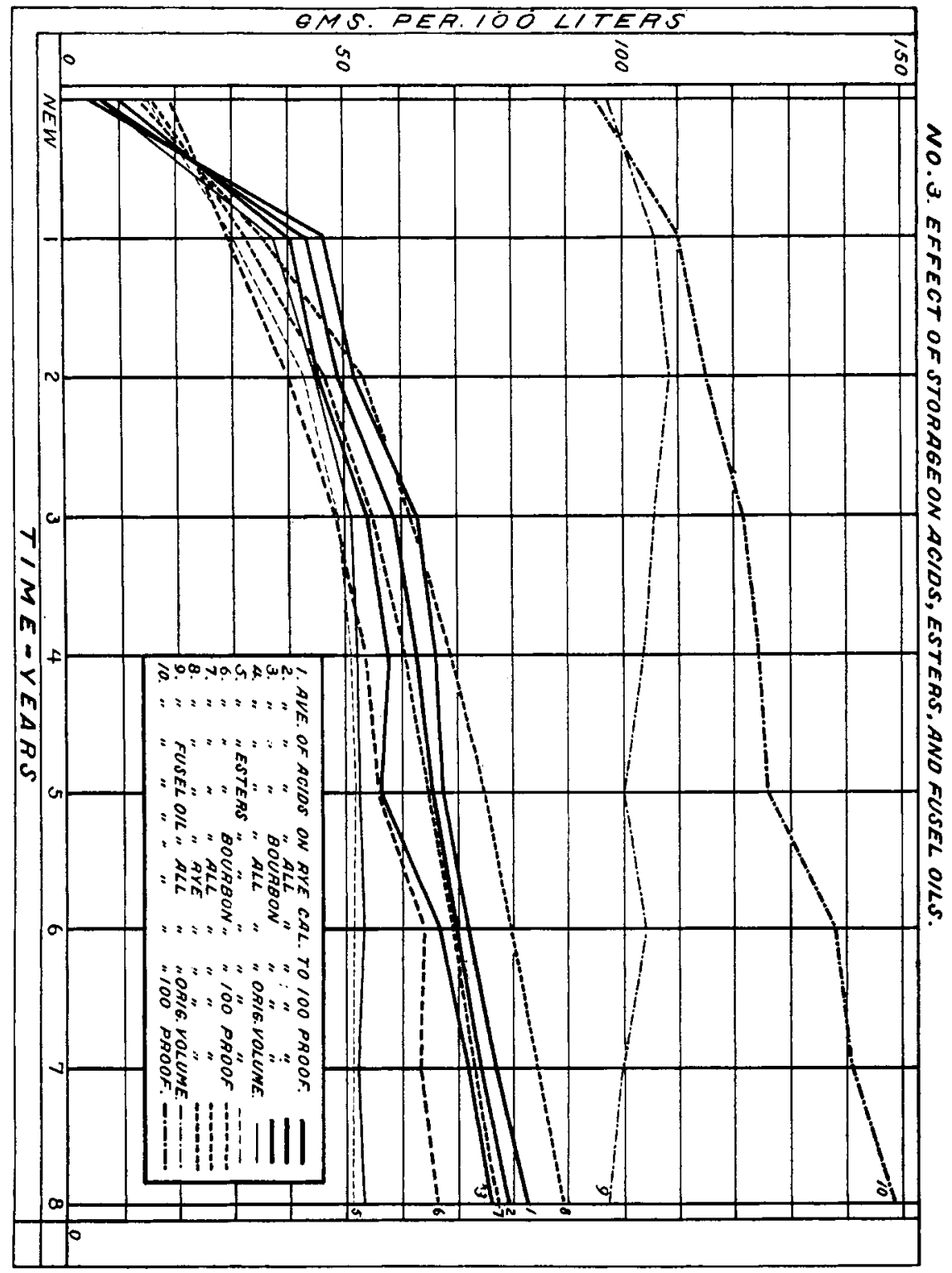

ters $6 r$, and they remain practically the same during the next four years. This shows that these two substances gradually approach an equilibrium, which they reach about the fourth year, and which does not change afterwards.

This point will be more fully discussed under chart $\mathrm{V}$, showing the relationship of the acids and esters for a number of years. 
NO. 4. RELATIONSHIP OEVELOPEO ON STORAGEIN 4 VEAR OLO GOODS.

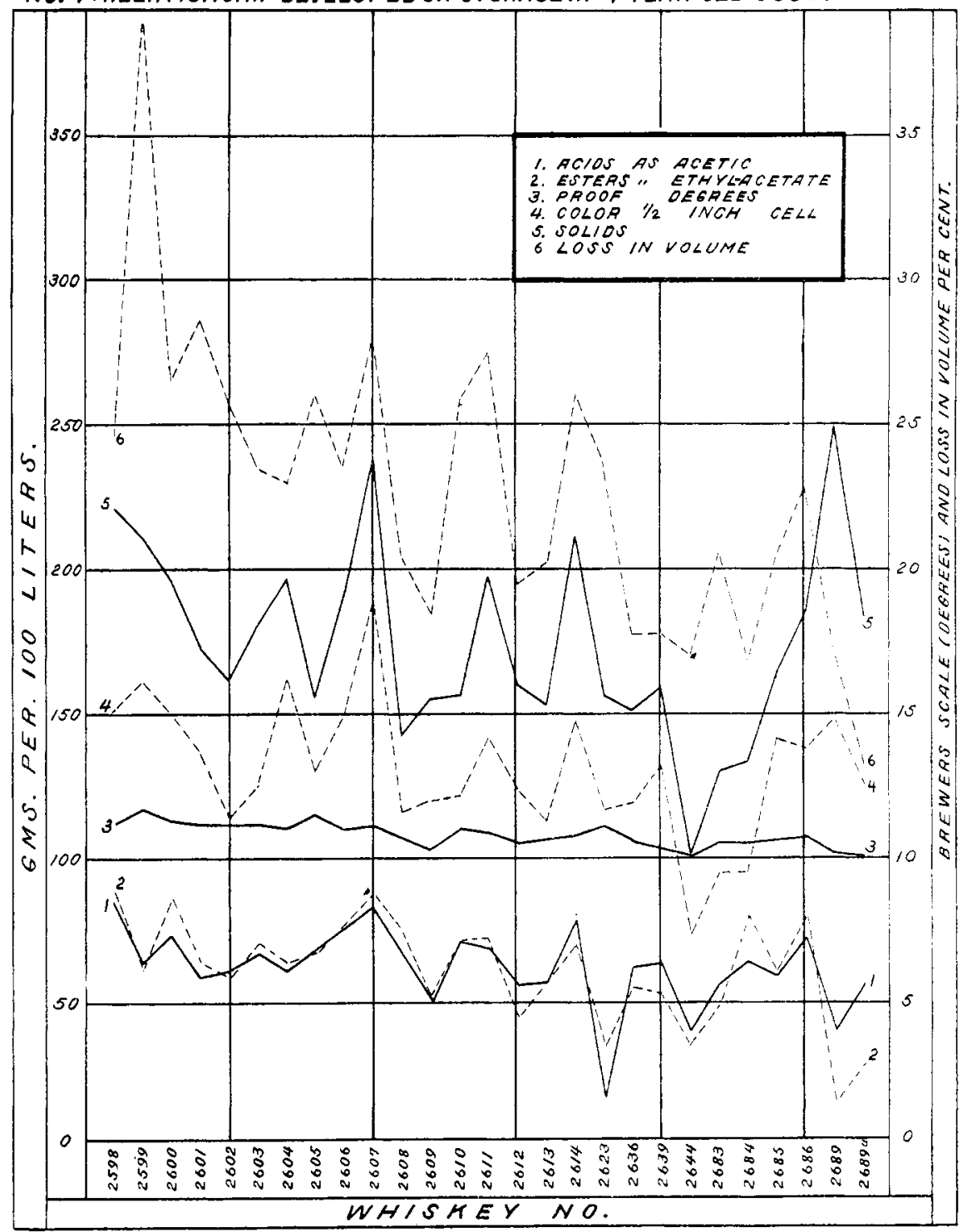

Chart IV, plotted from the results obtained on the four-year samples, shows the relationship of the various products present to each other and to the change in volume and the increase in proof.

Perhaps the most striking point brought out is the close relationship between the acids and the esters, the two lines following the same course across the sheet in the most remarkable way. But when we consider 
the dependence of one on the other this is not so strange. It is apparent from this, as well as from Chart I, giving the averages, and from Chart $\mathrm{V}$, on which are plotted the acids and esters, that these two substances reach an equilibrium, at the end of three or four years, as previously stated, which relationship does not change during longer storage. The meaning of this point will be discussed later.

This chart also gives the maxima and minima found for these samples, and in many cases shows how they may be explained by the loss in volume and increase in proof.

It also calls our attention to the fact that whereas when a whiskey has been matured in wood, and has a low acid content, we should expect to find a small amount of esters, it is shown in the new spirit and that when a year old this relationship does not hold, the two substances not having yet reached an equilibrium. This point may be of value in detecting young whiskies.

The color, solids, and concentration lines show a very close relationship, as might be expected, as they are dependent one on the other.

A study of all the lines reveals a marked relation among them. A high color, high solids, and high concentration are generally accompanied by high acids and esters, and low color and solids go with low acids and esters, which is a fact that will be of value in judging the purity of whiskies found on the market.

In fact, a study of the relationships found in these whiskies will be of great value in the determination of the adulteration of commercial whiskies. The range of variation that may take place in whiskies stored under different conditions is also shown by this chart.

Chart $\mathrm{V}$ shows the changes taking place in the acids and esters, using the results obtained on the new spirits, and those one, two and eight years old.

The great irregularity in the amounts of these substances in the new spirit, and the seeming lack of any relation, are at once noted; the acids, however, in every case, are lower than the esters, but at the end of the first year this has changed to a great degree, only seven samples showing higher esters than acids. The average of the esters, as shown in Chart III, is 9 grams per Ioo liters below that of the acids.

At the end of the second year the esters are on the average 7 grams below the acids, and by the end of the fourth year the acids and esters average the same, retaining this relation up to the eighth year, when the experiment ceased.

This shows that in the aging process the acids are formed more rapidy at first than the esters, but later the esters form more rapidly, so that by the end of the fourth year they are present in about the same amounts, and remain the same during storage. The fact that this equilibrium is 


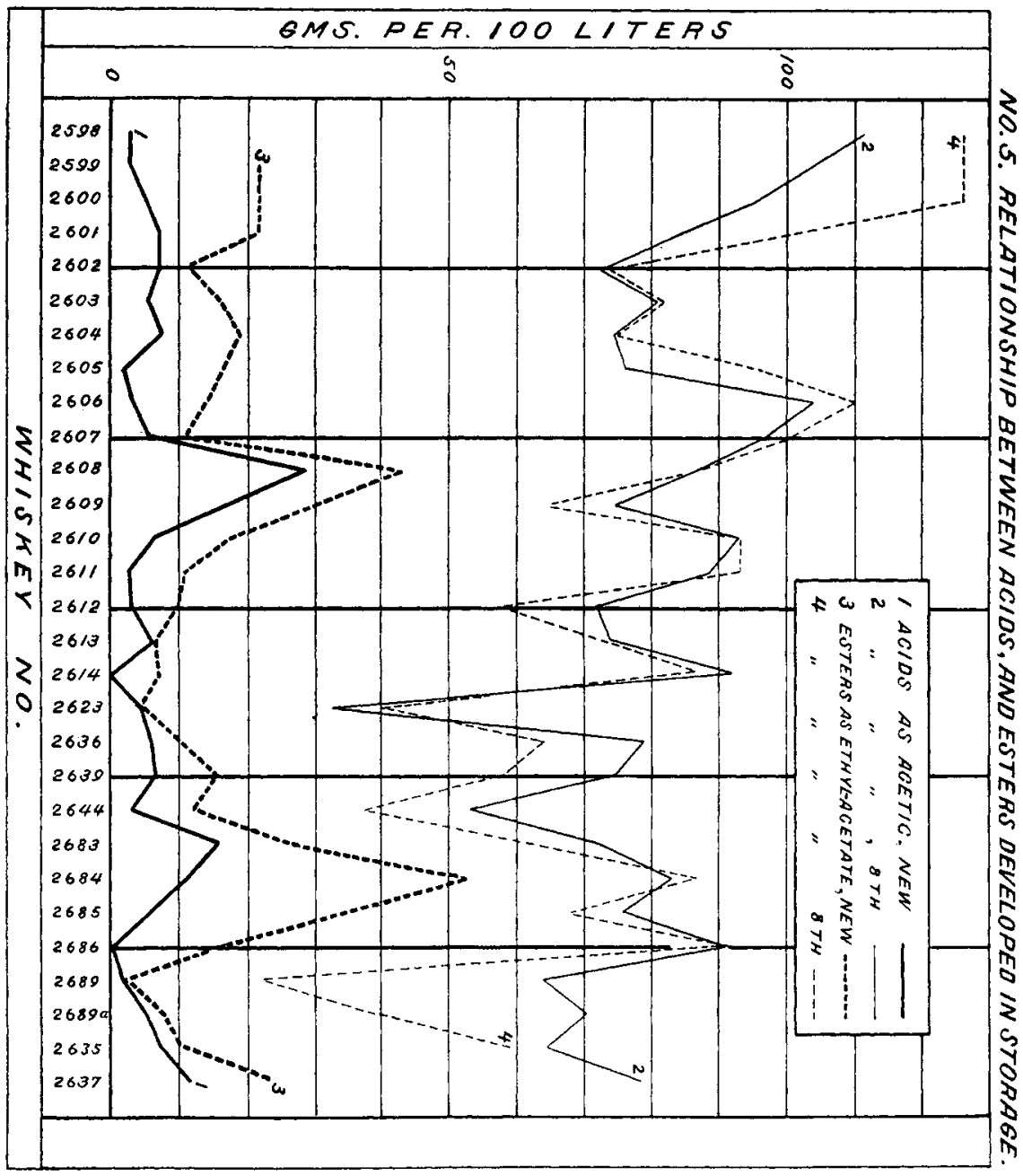

reached at the end of three or four years indicates that there is some very close relation between the equilibrium of the acids and esters and the ripening of the whiskey. This is, of course, only one of the factors in the aging, but it seems probable that, when this condition of equilibrium is reached, the whiskey is matured in so far as the acids and esters contribute to that condition.

The chart also calls attention to some very abnormal samples, for instance, Nos. 2623 and 2689 , both of which were aged in very cool warehouses, which fact probably explains in a degree the cause of their abnormalities. 


\section{Effect of Aging in Charred and Uncharred Packages.}

In order to determine the effects of the material extracted from the barrel in the process of aging on the flavor of the whiskey, especially the difference between charred and uncharred packages, two samples of sour mash Bourbon whiskey, which had been produced by the same distiller from very similar mashes, as shown by the table below, and distilled in the same general type of still, were taken for comparison.

\begin{tabular}{|c|c|c|c|c|}
\hline $\begin{array}{l}\text { Composition } \\
\text { of mash. }\end{array}$ & $\begin{array}{l}\text { Whisky in } \\
\text { uncharred } \\
\text { package. } \\
\text { Pounds. }\end{array}$ & Per cent. & $\begin{array}{l}\text { Whisky in } \\
\text { charred } \\
\text { package. } \\
\text { Pounds. }\end{array}$ & Per cent. \\
\hline Malt $\ldots \ldots \ldots \ldots \ldots$ & . 6048 & 10.9 & 4144 & II .O \\
\hline Rye.... & 4704 & $8 \cdot 5$ & 2688 & $7 \cdot 4$ \\
\hline Corn $\ldots \ldots \ldots \ldots \ldots \ldots \ldots$ & 45024 & 80.6 & 30800 & $8 \mathrm{I} .6$ \\
\hline
\end{tabular}

The samples had both been stored the same leingth of time in a warehouse and practically the only difference in the two products was in the kind of packages in which they were stored. The flavor, however, of the two whiskies was entirely different: the whiskey stored in the uncharred package lacked almost entirely the flavor so characteristic of American whiskies, having more of the flavor of Scotch or Irish whiskey, but without, of course, the smoky flavor of the Scotch, and was considered a very good-flavored whiskey by several persons who had a taste for such whiskies; the whiskey aged in the charred wood had the strong aromatic flavor which is so characteristic. In fact, the products are entirely different types of whiskey, showing that the method of aging is the important factor.

A study of the analytical data obtained, given in the following table, shows that there is a remarkable similarity in the amount of esters and acids and fusel oils in the two samples at the same age.

\begin{tabular}{|c|c|c|c|c|c|c|c|c|c|}
\hline \multirow[b]{2}{*}{ Serial number. } & \multirow[b]{2}{*}{ Proof. } & \multirow{2}{*}{$\begin{array}{c}\text { Color } \\
\text { in } \\
\text { y"n }\end{array}$} & \multirow{2}{*}{$\begin{array}{c}\text { Color } \\
\text { insolu } \\
\text { ble in } \\
\mathrm{H}_{2} \mathrm{O} . \\
\text { Per } \\
\text { cent. }\end{array}$} & \multicolumn{6}{|c|}{ Grams per 100 liters. } \\
\hline & & & & $\begin{array}{c}\text { Solids. } \\
\text { Per } \\
\text { cent. }\end{array}$ & $\begin{array}{c}\text { Acids. } \\
\text { Per } \\
\text { cent. }\end{array}$ & $\begin{array}{c}\text { Esters. } \\
\text { Per } \\
\text { cent: }\end{array}$ & $\begin{array}{c}\text { Alde- } \\
\text { hydes. } \\
\text { Per } \\
\text { cent. }\end{array}$ & $\begin{array}{l}\text { Fur- } \\
\text { furai. } \\
\text { Per } \\
\text { cent. }\end{array}$ & $\begin{array}{c}\text { Fusel } \\
\text { oil. } \\
\text { Per } \\
\text { cent. }\end{array}$ \\
\hline uncharred ath & 104.0 & $4 \cdot 5$ & . & 96.0 & 58.8 & 66.0 & 8.0 & 1.2 & Ior.o \\
\hline 35 , charred, $4^{\text {th }}$ year. & 105.0 & 9.0 & . & I 55.0 & 63.6 & $48 \cdot 4$ & 10.0 & 0.8 & 99.0 \\
\hline 27, uncharred, 8th year & 110.9 & $7 \cdot 0$ & 23 & 160.0 & 81.6 & $84 \cdot 5$ & 10.8 & I. 5 & II 2.0 \\
\hline 35 , charred, 8th year. & I10.0 & 13.5 & $6 I$ & 181.0 & 81.6 & 65.6 & 13.2 & 1.2 & 125.0 \\
\hline
\end{tabular}

The main difference between the two samples is in the amount of color and solids, and the composition of these solids and color, as shown by their solubility in water. The solids and color in the whiskey from the uncharred package are very much more soluble in water, being less of the resinous nature shown in the solids from the charred package, and in this respect it is very much like the Scotch whiskies which are aged in uncharred wood.

The resinous or oily nature of the solids in the whiskey from the charred 
package is also shown in the whiskey itself, the foam being much more oily and remaining much longer than the foan on the whiskey from the uncharred package.

The actual amount of solids in the eight-year old goods is not so very different in the two samples, but the amount of color in the uncharred is only 7.0 , as against 13.5 in the charred, and only 23 per cent. of the color in the uncharred is insoluble in water, while $6 \mathrm{I}$ per cent. of the color in the charred is insoluble, showing a wide difference in composition. As stated before, the whiskies are remarkably similar as far as the acids, esters, aldehydes, furfural and fusel oil are concerned, and the chief difference is in extractive matter.

The esters and acids in both have reached their equilibrium, and the maturity of the two samples, as far as these constituents are concerned, has been attained, but the marked difference in the flavor is due, without question, to the matter extracted from the barrels.

In order to determine the flavor due to the extractive matters, 100 cc. of each sample were taken and evaporated to $5 \mathrm{cc}$, , so that as much as possible of the esters and aldehydes and other volatile substances would be driven off.

To this residue were added a few cubic centimeters of water. The odor of the residue from the charred package was very strong, having the peculiar aroma of American whiskey. The taste of this residue was also very strong, being both resinous and astringent, and there can be no doubt that these materials, which are left in the residue, make up, to a great extent, the peculiar aroma of American whiskey, as distinguished from Irish or Scotch whiskey.

The residue from the uncharred package has a somewhat similar odor, and a slightly sour, astringent taste, entirely different from that of the charred package. This great difference in the flavor and odor of the residues from charred and uncharred packages is to a great degree the difference in the flavor and odor of the matured whiskey.

Whether all of these solids come from the package has not been proved, as part of these solids may come from the oxidation of some of the volatile oils which are distilled over, rendering them non-rolatile, but a sample containing only traces of fusel oil, which had been produced with a rectifying still hy which practically all of these oils are removed, and which had been aged in charred wood, was examined, and the solids prepared as above, had practically the same flavor. This at least indicates that the peculiar flavor which characterizes Anerican whiskies, as compared with Scotch and Irish whiskies, is largely due to the kind of package in which they are aged.

It cannot be considered, however, that the acids, esters, and other ingredients do not enter into the flavor of the matured product, but the 
peculiar flavor of American whiskies which distinguishes thein from other whiskies is undoubtedly derived from the extractive matter from the charred wood in which they are stored while aging.

This fact is brought out by sample 2612 , which was alcohol aged in wood, and lacked the taste of the whiskies produced in other type of stills, in which little rectification takes place.

\section{Flavor.}

It must be remembered that the flavor of these whiskies was determined at the same time, when all were of the same age, about nine yearsthe new spirit having been this length of time in bottle--and when we consider that it still had the odor of new whiskey, and showed no development of acids and esters, but was still raw spirit, it seems proved that none of the maturing processes take place in glass, and that whiskey after it is bottled will not improve.

In fact, none of the changes that take place in the wood occur in the bottled sample. Take, for instance, the year-old samples which have a very considerable amount of color and solids, and yet do not, in the eight years in which they are in glass, change as to solubility, like the solids and color of whiskey kept in wood. In fact in no way was any change indicated in the samples in glass.

No effort was made to judge when a certain whiskey had matured. The only comparison made was of the new spirit, the spirit four years in wood, and that eight years in wood, and then only in a general way to see whether the four- and eight-year old goods could be considered good whiskies, and whether the new spirit could in any way be said to have improved. In no case could the new spirit be said to be anything but raw spirits with the new whiskey flavor, not to be compared with the spirit one-year old.

The judging of the flavor and taste of the whiskies was done by a special gauger of the Iureau of Internal Revenue, who had a long experience in deciding between straight whiskies and compound whiskies, and has shown his ability to detect the difference. The samples were submitted without name for an unprejudiced decision. The results will not be given in detail, as all of the matured samples would doubtless be considered good whiskey, and a judgment as to quality is very difficult and unsatisfactory, and unnecessary for this paper.

Special attention was given to a few samples which were peculiar: No. 2612, which was practically cologne spirits aged in wood, No. 2623, which was abnormal in content of acids and esters, No. 2689, which was abnormal in content of esters, and Nos. 2625 and 2627 , which were stored in uncharred packages.

The judgment of No. 2612 was that it had the peculiar aroma of Amer- 
ican whiskey, but had the taste of spirits, and little difference could be noted between the two-year old sample and the eight-year old.

The judgment of No. 2623 was that it was a good flavored whiskey, but with very light body.

No. 2689 was pronounced a good flavored whiskey.

Nos. 2625 and 2627 , the samples aged in uncharred packages, were both called immature whiskey, even when the eight-year old sample was tested. But this judgment was based on a comparison with those aged in charred packages.

On that basis the eight-year old sample was thought to be about i to 2 years old. This shows what a marked effect the storage in charred wood has upon the flavor of the whiskey.

\section{Conclusions.}

1. There are important relationships among the acids, esters, color, and solids in a properly aged whiskey, which will differentiate it from artificial mixtures and from young spirit.

2. All of the constituents are undergoing changes as the aging process proceeds, and it is evident that the matured whiskey is the result of these combined changes.

3. The amount of higher alcohols increases in the matured whiskey only in proportion to the concentration.

4. Acids and esters reach an equilibrium, which is maintained after about three or four years.

5. The characteristic aroma of American whiskey is derived almost entirely from the charred package in which it is aged.

6. The rye whiskies show a higher content of solids, acids, esters, etc., than do the Bourbon whiskies, but this is explained by the fact that heated warehouses are almost universally used for the maturing of rye whiskies, and unheated warehouses for the naturing of Bourbon whiskies.

7. The improvement in flavor of whiskies in charred packages after the fourth year is due largely to concentration.

8. The oily appearance of a matured whiskey is due to material extracted from the charred package, as this appearance is almost lacking in whiskies aged in uncharred wood.

9. The "body" of a whiskey, so-called, is due largely to the solids extracted from the wood.

[CONTRibltion from the Chemical Laboratory OF the University of NoRth CAROLINA.]

THE CONDENSATION OF CHLORAL WITH PRIMARY AROMATIC AMINES. II.

A number of condensation products of chloral with primary aromatic 